\title{
Delivery of retinoic acid to LNCap human prostate cancer cells using solid lipid nanoparticles
}

\author{
Mushfiq H. Akanda ${ }^{1}$, Rajeev Rai ${ }^{1}$, Ian J. Slipper ${ }^{1}$, Babur Z. \\ Chowdhry $^{1}$, Dimitrios Lamprou ${ }^{2}$, Giulia Getti ${ }^{1}$, Dennis Douroumis ${ }^{1 *}$
}

${ }^{1}$ Faculty of Engineering and Science, University of Greenwich, Medway

Campus, Chatham Maritime, Kent ME4 4TB, UK

${ }^{2}$ Strathclyde Institute of Pharmacy and Biomedical Sciences, University of

Strathclyde, The John Arbuthnott Building, Glasgow G40NR, Scotland

\footnotetext{
* Corresponding author, Department of Pharmaceutical, Chemical \& Environmental Sciences, Faculty of Engineering and Science, University of Greenwich, Medway Campus, Chatham Maritime, Kent ME4 4TB, UK. Tel.: +44 (0) 208331 8440; Fax: +44 (0) 208331 9805. E-mail address: D.Douroumis@gre.ac.uk (D. Douroumis).
} 


\begin{abstract}
In this study retinoic acid (RTA) loaded solid lipid nanoparticles (SLNs) optimized by tuning the process parameters (pressure/temperature) and using different lipids to develop nanodispersions with enhanced anticancer activity. The RTA-SLN dispersions were produced by high-pressure homogenization and characterized in terms of particle size, zeta potential, drug entrapment efficiency, stability, transmission electron microscopy (TEM), atomic force microscopy (AFM), X-ray diffraction (XRD) and in vitro drug release. Thermal and X-ray analysis showed the RTA to be in the amorphous state, whilst microscopic images revealed a spherical shape and uniform particle size distribution of the nanoparticles. Anticancer efficiency was evaluated by incubating RTA-SLNs with human prostate cancer (LNCap) cells, which demonstrated reduced cell viability with increased drug concentrations $(9.53 \%$ at $200 \mathrm{ug} / \mathrm{ml}$ ) while blank SLNs displayed negligible cytotoxicity. The cellular uptake of SLN showed localization within the cytoplasm of cells and flow cytometry analysis indicated an increase in the fraction of cells expressing early apoptotic markers, suggesting that the RTA loaded SLNs are able to induce apoptosis in LNCap cells. The RTA-SLN dispersions have the potential to be used for prostate anticancer treatment.
\end{abstract}

Key Words:

Retinoic acid

Solid Lipid Nanoparticles,

Cytotoxicity

Prostate cancer

Flow cytometry 


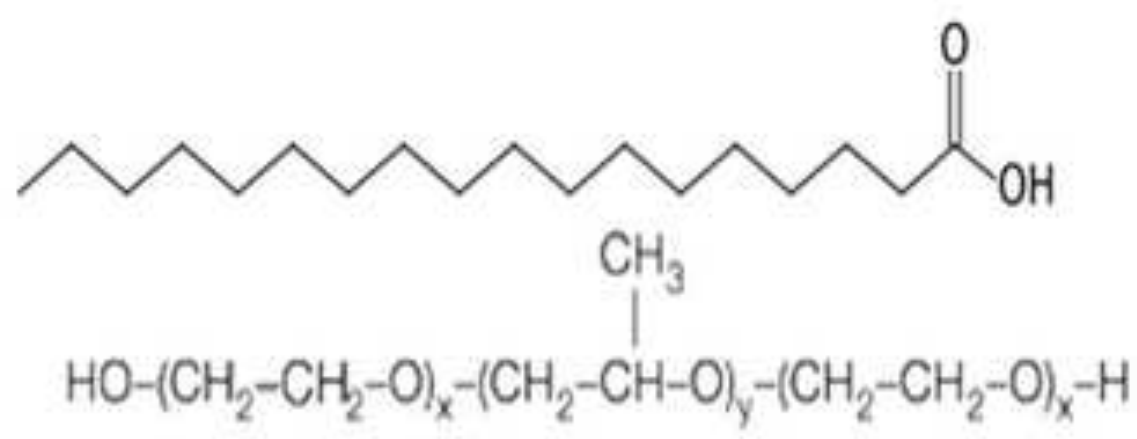

\section{High pressure homogenization}
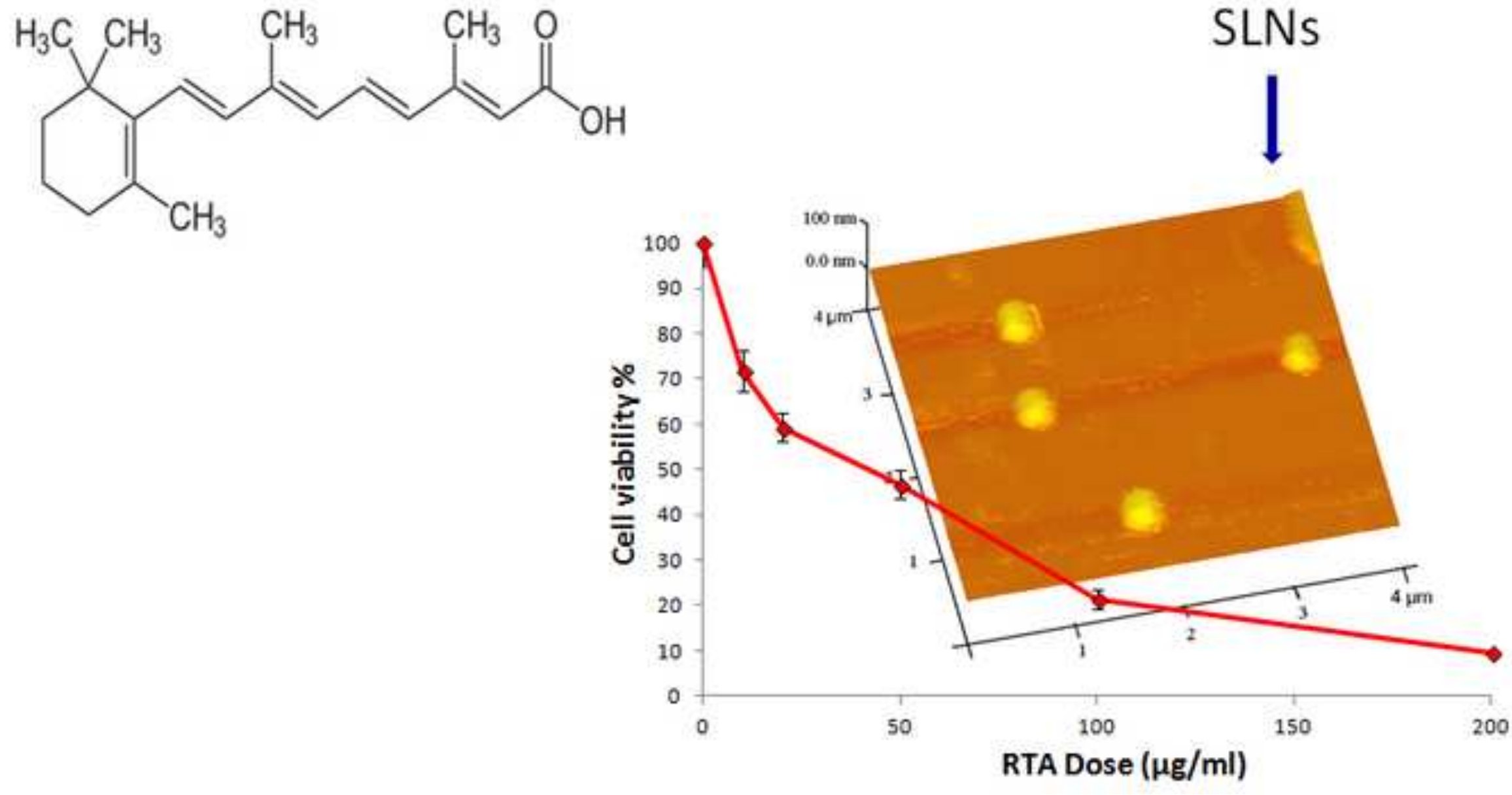


\section{Introduction}

Solid lipid nanoparticles (SLNs) have attracted increasing attention as a promising colloidal carrier system, especially for lipophilic drugs (Chen et al., 2001; Mehnert et al., 2001; Müller et al., 2004; Castelli et al., 2005). SLNs are spheres or platelets in the submicron size range (mainly between 150 and $300 \mathrm{~nm}$ ). These are made of lipids, which are solid at room and body temperature and dispersed in an aqueous medium (Müller et al., 2011; Battaglia and Gallarate., 2012). SLNs are composed of a high melting point lipid as a solid core, which is coated by surfactants. Therefore, lipophilic drugs can be efficiently incorporated in the lipid core of SLNs. SLNs consist of a solid core rather than a fluid core, such as liposomes and emulsions, which facilitates prolonged and controlled release of drugs (Mehnert et al., 2001). SLN incorporated drugs are protected against chemical degradation (Lim et al., 2002). The production of SLNs is easier to scale up compared with other drug delivery systems such as polymeric nanoparticles or liposomes (Mueller et al., 2000). The advantages of SLNs have driven numerous studies with various applications, particularly for parenteral administration of drugs. Typical parenteral SLNs applications include intraarticular to intravenous peroral administration (Muller et al., 2000) increased drug solubility (Yang et al., 2014) and pulmonary delivery (Yang et al., 2012). Studies performed by Gasco et al., revealed that intravenously administered SLNs display prolonged drug plasma levels. Moreover, a much lower uptake by liver and spleen macrophages occurs due to the low surface hydrophobicity of SLNs avoiding the absorption of any blood proteins (Gasco et al., 1993; Goppert and Muller, 2005).

Retinoic acid (RTA) is a promising anticancer agent, which has been investigated in chemoprevention and treatment of cancer (Orlandi et al., 2002; Carneiro et al., 2012). The anticancer properties of RTA are achieved by binding to retinoic acid receptors or retinoid X receptors present on the nuclear membrane of cancer cells, leading to the induction of growth inhibition, differentiation or apoptosis in these cells (Fang et al., 2002). RTA has already been used in clinical trials where RTA was given to cancer patients by oral administration, though the RTA concentration in blood circulation gradually decreases after long-term oral treatment. This phenomenon might occur due to the induced cytochrome P-450-dependent metabolism of RTA (Muindi et al., 1992). The poor aqueous solubility of RTA can be a major drawback for its parenteral administration. However, the incorporation of RTA in lipid-based carriers such as SLNs could be an attractive means to overcome such solubility 
limitations. The aim of this study was to develop stable RTA-SLN formulations with improved cellular uptake and anticancer efficacy in prostate LNCaP cancer cells.

\section{Materials and methods}

\subsection{Materials}

Retinoic acid was purchased from TCI (UK). Precirol ${ }^{\circledR}$ ATO 5 (PR) was purchased from Gattefosse. Poloxamer 188 (P188) was kindly donated by BASF (Ludwigshafen, Germany). LNCap cell line was purchased from the American Type Culture Collection (ATTC, Manassa, Virginia, USA). Stearic acid (SA), tristearin (TS), trilaurin (TL), Dulbecco's modified Eagle's medium (DMEM), thiazolyl blue tetrazolium bromide (MTT), L-glutamine, penicillin, streptomycin, heat inactivated fetal bovin serum (FBS) and trypsin were purchased from Sigma-Aldrich (UK). Rhodamine was purchased from Avanti polar lipids (USA). PE Annexin V Apoptosis Detection Kit I containing 7AAD as vital stain was obtained from BD Biosciences, UK. All the other chemicals and solvents used were of analytical or highperformance liquid chromatography (HPLC) grade.

\subsection{Preparation of SLNS}

SLNs were prepared by high pressure homogenization. In brief, appropriate amounts of lipids e.g., SA and P188 were accurately weighed and heated above the melting point of the lipid. For RTA loaded SLNs the drug was separately dissolved in ethanol (3 ml) and then added to the molten lipid. The drug containing the melted lipid was dispersed in a hot aqueous phase and homogenized with an UltraTurrax T25 (IKA®- WERKE GMBH, Staufen, Germany) homogenizer to form a pre-emulsion. The coarse dispersion produced was then transferred and homogenized in a Micro DeBee (South Easton, MA, USA) high pressure homogenizer at $15,000 \mathrm{psi}$ for $7 \mathrm{~min}$ at $70^{\circ} \mathrm{C}$. The hot nano-dispersions were left to cool and allow the lipid to crystallize by forming lipid nanoparticles with a solid matrix.

\subsection{Particle size analysis and zeta potential}

The particle size distribution and zeta potential of the produced preparations were determined by dynamic light scattering photon correlation spectroscopy (PCS) using a Malvern Zetasizer Nano-ZS (Malvern, UK). The dispersion were adequately diluted with distilled water and measured in triplicate. The z-average and the PDI were applied for the evaluation of the particle size. The particle size range determined was $0.6 \mathrm{~nm}-6 \mu \mathrm{m}$. 


\subsection{Atomic force microscopy}

For AFM experiments, $3 \mu \mathrm{L}$ of RTA loaded and unloaded SLN were deposited onto a freshly cleaved mica surface ((G250-2 mica sheets 1" x 1" x 0.006"; Agar Scientific Ltd, Essex, UK), and left to dry for $1 \mathrm{~h}$ before AFM imaging. The images were obtained by scanning the mica surface, in air, under ambient conditions using a PeakForce QNM Scanning Probe Microscope (Digital Instruments, Santa Barbara, CA, USA; Bruker Nanoscope analysis software Version 1.40). The AFM measurements were obtained using ScanAsyst-air probes, and the spring constant $(0.67 \mathrm{~N} / \mathrm{m}$; nominal $0.4 \mathrm{~N} / \mathrm{m})$ and deflection sensitivity were calibrated, but not the tip radius (a nominal value of $2 \mathrm{~nm}$ was used). Surface roughness $(\mathrm{Ra})$ values were determined by entering surface scanning data into a digital levelling algorithm (Bruker Image Analysis Nanoscope Analysis software V 1.40). AFM images were collected from two different samples by random spot surface sampling (at least five areas).

\subsection{Transmission electron microscopy (TEM)}

The shape and size of the SLN dispersions were investigated by TEM. Before analysis, the samples were diluted 1:2 and stained with $2 \%(\mathrm{w} / \mathrm{v})$ phosphotungstic acid for $30 \mathrm{~s}$ and placed on copper grids with films for observation. After 1 min of absorption, excess liquid was blotted off with filter paper. The dried specimens were examined by using a Hitachi SH 8030 microscope. The electron beam was accelerated with a voltage of $10 \mathrm{kV}$. The SEM images were captured using a Gatan, Inc. ORIUS SC200 CCD Camera. Several magnification levels were used.

\subsection{Lyophilisation of SLNS}

The empty and RTA loaded SLN dispersions were freeze-dried without the addition of cryoprotectants using an Advantage freeze-dryer (Biopharma process systems, UK). The lyophilisation process was carried out by cooling the samples from $20^{\circ} \mathrm{C}$ to $-50^{\circ} \mathrm{C}$ at $0.5^{\circ} \mathrm{C} / \mathrm{min}$ under atmospheric pressure and samples were held at $-50^{\circ} \mathrm{C}$ for $2 \mathrm{hr}$. After $2 \mathrm{hr}$ the pressure was reduced to $30 \mathrm{mTor}$, the sample plates were heated to $-10^{\circ} \mathrm{C}$ and the samples were dried under the aforementioned conditions for $48 \mathrm{hr}$. Afterwards the plates were slowly heated at $0.2^{\circ} \mathrm{C} / \mathrm{min}$ to $20^{\circ} \mathrm{C}$ and the pressure reduced to $10 \mathrm{mTor}$ for $2 \mathrm{hr}$ to remove residual water 


\subsection{X-ray powder diffraction}

Samples of bulk RTA, empty SLNs and RTA-SLN formulations were evaluated using a Bruker D8 Advance (Coventry, UK) diffractometer in theta-theta mode, $\mathrm{Cu}$ anode at $40 \mathrm{kV}$ and $40 \mathrm{~mA}$, parallel beam Goebel mirroe, $0.2 \mathrm{~mm}$ exit slit, Lynxeye position sensitive detector (PSD) with a $3^{\circ}$ opening and LynxIris at $6.5 \mathrm{~mm}$, sample rotation at $15 \mathrm{rpm}$. The sample was scanned from $2^{\circ}$ to $40^{\circ} 2 \theta$ with a step size of $0.02^{\circ} 2 \theta$ and a counting time of $0.2 \mathrm{~s}$ per step; 176 channels active on the PSD making a total counting time of $35.2 \mathrm{~s}$ per step.

\subsection{Differential scanning calorimetry (DSC)}

DSC measurements of bulk RTA, empty SLNs and RTA-SLN formulations were conducted using a Mettler Toledo DSC 823 instrument (Schwerzenbach, Switzerland). Approximately, 3-5 mg samples were accurately weighed in standard aluminium pans. An empty pan was used as a reference. A scan rate of $10^{\circ} \mathrm{C} / \mathrm{min}$ was employed to heat the samples from 20 to $220^{\circ} \mathrm{C}$. Analysis was performed under a nitrogen purge $(60 \mathrm{~mL} / \mathrm{min})$. Calorimetric parameters were analysed using STARe Software.

\subsection{Determination of encapsulation efficiency}

The drug loading (DL) and encapsulation efficiency (EE) was estimated according to Potta et al., (2011). In brief, $1 \mathrm{ml}$ of SLN nano-suspension was centrifuged at 40,000 rpm, for 30 min at $25^{\circ} \mathrm{C}$. The pellets of RTA loaded SLNs were then dissolved in acetonitrile and the absorbance of the dissolved pellets were measured after appropriate dilutions. The DL and encapsulation efficiency (EE\%) were calculated by using Equations (1) and (2), respectively.

$$
\begin{aligned}
& \text { Drug loading }=\frac{\text { Amount of RTA in SLNS }}{\text { Amount of SLNS }} \times 100 \\
& \text { Encapsulation efficiency }=\frac{\text { Drug loading }}{\text { Theoretical drug loading }} \times 100
\end{aligned}
$$

\subsection{Drug release properties of RTA-SLNs}

A dispersion of SLNs $(1 \mathrm{ml})$ was transferred into a cellulose dialysis bag (molecular weight cut-off: $10,000 \mathrm{Da}$ ), which was then suspended in a beaker containing a mixture of 
double-distilled water and ethanol (50:50, v/v) as the dissolution medium (Liu et al., 2007). The beaker was then placed in a shaker bath at a temperature of $37^{\circ} \mathrm{C}$. At various time intervals the dissolution medium $(20 \mathrm{ml})$ was replaced and the drug content was analysed by UV spectroscopy using a $\lambda \max$ of $340 \mathrm{~nm}$.

\subsection{Cytotoxicity tests}

LNCap prostate cancer cell lines were cultured in DMEM medium supplemented with $10 \%$ serum, $1 \mathrm{X} \mathrm{L-glutamine/penicillin} \mathrm{streptomycin} \mathrm{(Sigma,} \mathrm{UK}$ ) at $37^{\circ} \mathrm{C}$ and $5 \% \mathrm{CO}_{2}$. The culture medium was changed every three days. The cytotoxicity of RTA loaded SLNs and pure RTA (dissolved in ethanol) was determined in the LNCap prostate cancer cell line using the MTT assay. Cells were seeded in a 24 well flat-bottom plates at a cell density of $1 \times 10^{6}$ cells/well in $1 \mathrm{ml}$ DMEM and incubated for $24 \mathrm{hr}$. The SLN formulations, (empty, loaded), pure RTA and ethanol were then added into the 24 well plates at various concentrations for different incubation times $(24,48 \mathrm{hr})$. Pure RTA was solubilized in ethanol $(10 \mathrm{mg} / \mathrm{ml})$ and incubated at concentrations of $10,20,50,100,200 \mu \mathrm{g} / \mathrm{ml}$. At the end of the incubation time $100 \mu \mathrm{L}$ of MTT solution ( $5 \mathrm{mg} / \mathrm{ml}$ ) was added to each well and incubated at $37^{\circ} \mathrm{C}$ for another $2 \mathrm{hr}$. The culture medium was then discarded and $200 \mu \mathrm{l}$ of acidified isopropanol was added in order to dissolve the MTT formazan crystals. A total of $100 \mu \mathrm{L}$ of the dissolved MTT formazan crystals were transferred into a 96 well flat-bottom plate and the absorbance read at $492 \mathrm{~nm}$ using a microplate reader. Controls included non-treated cells and ethanol (2\%) for the pure drug cytotoxicity assay. Blank (unloaded) and loaded SLN formulations were incubated and the cytotoxicity of blank and loaded SLN formulations were also tested on LNCap cells. Concentrations of blank SLNs were kept between 0.18, 0.37, 0.73, 1.11, 1.47 and $2.94 \mathrm{mg} / \mathrm{ml}$ while loaded SLN formulations had drug (RTA) concentrations of 12.5, 25, $50,75,100$ and $200 \mu \mathrm{g} / \mathrm{ml}$.

\subsection{Cellular uptake by fluorescent microscopy}

Samples for microscopic analysis were prepared via seeding $2 \times 10^{4}$ cells/well on cover slips in a 24 well flat-bottom plate. Empty rhodamine $(10 \mu \mathrm{g} / 10 \mathrm{mg}$ of lipid $)$ stained SLN formulations were incubated with the cells for $24 \mathrm{hr}$ at various concentrations. The cell medium was discarded from the well after the incubation and washed three times with PBS; $1 \mathrm{ml}$ of $4 \%$ para-formaldehyde was added to each well to fix the cells on the cover slips and left in the dark for $15 \mathrm{~min}$. The para-formaldehyde was discarded from the well and cells were washed three times with PBS and then mounted on a glass slide using vectashield 
mounting medium containing DAPI. The cover slips were sealed on a glass slide with a nail polish and left to dry. Images were acquired using the Nikon ECLIPSE 90i overhead epifluorescent microscope attached to a Nikon digital camera (DS-Qi1Nc) and a computer running Nikon NIS-Elements Advanced Research software. The principal objective used for fluorescent imaging was an oil immersion CFI Plan Apochromat VC 60X N2 (NA1.4, WD $0 \cdot 13 \mathrm{~mm})$.

\subsection{In vitro apoptosis studies}

Apoptosis is a form of cell death that plays an important role during development, normal tissue homeostasis and is deregulated in many diseases, including cancer. The effect of apoptosis in LNCap prostate cancer cell lines was studied by flow cytometry. The early stages of apoptosis were detected following staining with a PE Annexin V Apoptosis Detection Kit I containing 7AAD as vital stain. The cells were seeded in 24 well flat-bottom plates at a cell density of $1 \times 10^{6}$ cells/well and incubated for $24 \mathrm{hr}$. SLN formulations, (empty, loaded) and pure RTA ( $25 \mathrm{ug} / \mathrm{ml})$ were added to each well. Untreated cells were used as negative controls. After $48 \mathrm{hr}$, cells were stained according to the manufacturer's protocol. Samples were analysed on by using a Accuri C6 flow cytometer for PE and 7AAD expression using a solid state blue laser with a $488 \mathrm{~nm}$ excitation spectrum and a detector of FL1 path with a 530/30 nm filter. A minimum of 10000-gated events was acquired from the cell population and data analysed using the Accuri C6 software. Cells were considered early apoptotic when PE positive and 7AAD negative; late apoptotic/early necrotic when PE positive and 7AAD positive. All experiments were performed in triplicate and repeated three times.

\subsection{Statistical analysis}

Results were expressed as means and standard deviation of the mean $(n=3)$. Statistical significance was also determined by t-test analysis for comparison between RTA loaded SLN and blank SLN formulations on the LNCap prostate cancer cell line. Differences were considered as significant when the $P$-value was $<0.05$ 


\section{Results and discussion}

\subsection{Particle size distribution and zeta potential}

The particle size and zeta potential of blank SLN's prepared by using various lipids was investigated by using the same lipid/surfactant ratios, as shown in Fig. 1 and Table 1. Laser diffraction analysis showed monomodal particle size distribution for all dispersions with sizes varying from $140-150 \mathrm{~nm}$ and polydispersity indexes of less than 0.22 . It is obvious that the nature of the lipid did not affect the particle sizes obtained for the SLNs. Similarly the zeta potential varied between -13 to $-19 \mathrm{mV}$, which is an indication of longterm stability as the higher zeta potential values indicate high electric charge on the surface of the SLNs, which induces strong repulsion forces between the particles preventing aggregation of the SLN dispersions (Yousefi et al., 2009). Indeed SLN dispersions were stable over six months with a slight increase in the particle size varying from $10-20 \mathrm{~nm}$ (data not shown).

RTA loaded SLNs of the same lipids were then prepared by high-pressure homogenisation at temperatures above the lipid melting point. However, during the process optimization it was observed that RTA-SLNs prepared with TL and PR were very unstable followed by drug precipitation within a few hours even at low drug loadings and thus further evaluation of RTA-SLNs with these two lipids was not possible. The loaded SLN dispersions prepared using TS and SA displayed far better stability and were further evaluated. RTASLNs prepared by using TS as the core lipid showed an average particle size of $255.9 \mathrm{~nm}$ and zeta potential of $-0.05 \mathrm{mV}$ but appeared to be unstable during long term stability. After six months, RTA recrystallization was observed and a second particle size peak at $3.3 \mu \mathrm{m}$ appeared, as shown in Fig. 2.

In contrast RTA-SLNs prepared by using SA as the core lipid showed a narrow monomodal particle size distribution with a $\mathrm{z}$-average diameter of $232.3 \pm 3.1 \mathrm{~nm}$ and a zeta potential of $-8.55 \pm 1.71 \mathrm{mV}$. As shown in Table 2 for the RTA loaded SLNs, a significant particle size increase was observed compared to the empty dispersions of the same lipids. This increase can be attributed to drug incorporation in the lipid nanospheres. In addition, RTA can cause significant increase in the viscosity resulting in an increase in particle size (Padhye et al., 2013).

\subsection{TEM and AFM morphology of SLNS}

Fig. 3 shows the TEM images of empty and RTA loaded SLNs, which appear to be spherical with a uniform particle size distribution. However, the SLN particle size in the 
TEM images appears to be between 50-60 nm, which is not in good agreement with the results obtained from the laser diffraction measurements. The reasons for the different particle sizes are due to the following two reasons: a) laser diffraction measures the "hydrodynamic diameter", which depends on the surface structure and not only on the particle "core" and b) for the TEM analysis, SLNs were stained with $2 \%(\mathrm{w} / \mathrm{v})$ phosphotungstic acid and subsequently dried which probably caused significant shrinkage of the lipid particles (Liu et al., 2007). For this reason AFM analysis, which can identify material properties at high resolution across a topographic image, was used to measure the SLN particle size. The overall particle diameter (estimated from the width of the peak at the baseline in section height profiles), for both empty and loaded SLN dispersions was estimated, as shown in Fig. 4. AFM analysis revealed spherical shaped nanoparticles with particle sizes of $117.8 \pm 11.0 \mathrm{~nm}$ and $169.8 \pm 21.0 \mathrm{~nm}$ for empty and loaded SLNs, respectively. The results obtained from the AFM imaging are similar to those from the laser diffraction measurements but with slightly smaller particle diameters. Again the reason for the observed smaller particle sizes is that SLNs were absorbed on the mica surface and underwent a drying process, which might have contributed to the size deformation (Jung et al., 2006). Moreover, in AFM studies there is a slight flattening of the SLNs which might also be responsible for the small difference between the diameter and the height of nanoparticles (Dubes et al., 2003).

\subsection{Physicochemical characterization}

The physical state of bulk materials, empty SLNs and RTA-SLNs was evaluated by XRD analysis. The empty and drug loaded dispersions were freeze-dried prior to the X-ray analysis. As shown in Fig. 5 the diffractograms of bulk SA and RTA displayed characteristic sharp peaks at $6.63^{\circ}, 11.1^{\circ}, 21.55^{\circ}, 24.15^{\circ}$ and $5.19^{\circ}, 13.52^{\circ}, 14.71^{\circ}, 15.56^{\circ}, 20^{\circ}, 20.8^{\circ}$, $22.81^{\circ}, 24.98^{\circ}, 24.98^{\circ}, 26.15^{\circ}, 27.23^{\circ} 2 \theta$ values, respectively which indicated the crystalline nature of both materials. Similarly P188 presented intensity peaks at $19.17^{\circ}, 23.36^{\circ} 2 \theta$ values. In Fig. 6 it is evident that the less ordered crystals of SA are present in both empty and loaded SLNs, where the majority of the intense peaks are not present. Likewise no high intensity peaks for RTA could be detected for the drug loaded SLNs suggesting that RTA is entrapped within the lipid core in an amorphous form (Mulik \& Monkkonen, 2010).

DSC thermal analysis of bulk SA, P88, RTA empty and loaded SLNs (Fig. 7) were conducted in order to further investigate the physical state of the colloidal lipid matrices. The 
bulk SA, P188 and RTA presented endothermic melting peaks at $69.82^{\circ} \mathrm{C}, 51.81^{\circ} \mathrm{C}$ and $167.80^{\circ} \mathrm{C}$, respectively. The SLN formulations showed two distinct melting endothermic peaks at $49^{\circ} \mathrm{C}$ and $66.49^{\circ} \mathrm{C}$, which are attributed to the surfactant and the lipid, respectively. The melting endotherms of empty SLNs appeared broad and less sharp compared to the bulk substances indicating an increased number of lattice defects (Siekmann et al., 1994). In addition, the melting peaks for both empty and loaded SLNs were shifted to lower temperatures indicating possible surfactant-lipid interactions (Padhye et al., 2013). For the RTA-SLNs the melting endotherm of RTA could not be observed suggesting, again, that the drug is in an amorphous state. However, RTA could have been completely solubilised in the lipid matrix due to the melting of drug in lipid (Padhye et al., 2013). In another study Mulik et al. (2012) proposed that these findings indicate the entrapment of RTA within the nanoparticles in a molecularly dispersed form.

\subsection{Determination of $E E$ and $D L$}

RTA-SLNs prepared either with SA or TST as the core lipid demonstrated EE of $90 \%$ suggesting very high drug entrapment in the nanoparticles. As RTA is very hydrophobic the stability of the drug within the lipid matrix is considered to be the key factor underlying drug encapsulation within the SLN (Chinsriwongkul et al., 2011). According to Tiyaboonchai et al., (2007) addition of surfactant in SLN dispersions plays a key role in enhancing the drug loading efficiency. In the developed SLN dispersions P188 was used as a surfactant, where the estimated DL observed was $6.2 \%$. Nevertheless, the DL was not very high as RTA started precipitating immediately when drug loading was increased.

\subsection{Drug release studies}

As shown in Fig. 8, RTA-SLN formulations presented a biphasic in vitro drug release pattern. A burst release was observed for the first $10 \mathrm{hr}$ where almost $40 \%$ of the drug was released, followed by sustained release for five days. The initial burst release characteristics indicates that some drug molecules were adsorbed on the surface of the SLN particles, while the sustained release pattern suggests drug diffusion from the core of the lipid matrix (Sun et al., 2013). According to Nayak et al., (2010) the drug release patterns from the lipid core depends greatly on the crystallinity of the lipid matrix. As shown from the XRD and thermal analysis results, RTA loaded SLN dispersions showed less ordered crystals of the lipid matrix, which facilitates faster drug elution from the SLNs. 


\subsection{Cytotoxicity of RTA and SLN formulations}

The cytotoxicity of bulk RTA, empty and RTA loaded SLN was evaluated in LNCaP prostate cancer cells by using the MTT assay. The RTA cyctotoxicity was extrapolated by comparison with alcoholic solutions and loaded SLNs. According to Serpe et al., (2004), SLNs can enter cancer cells, which should facilitate the anticancer activity of the drug when encapsulated in the lipid core.

RTA a potent anticancer agent showed strong cytotoxicity on LNCap cell lines at a concentration of $20 \mu \mathrm{g} / \mathrm{ml}$, where the cell viability was reduced to $59.3 \%$ after $24 \mathrm{hr}$ incubation (Fig. 9). The RTA cytotoxicity increased with the drug concentration and at $50 \mu \mathrm{g} / \mathrm{ml}$ the viability was reduced to $46.8 \%$ while a further increase to $200 \mu \mathrm{g} / \mathrm{ml}$ of RTA resulted in a cell viability of $9.5 \%$. This observation is in agreement with previous data on the effect of RTA on prostate cancer cells (McCormick et al., 1999).

As shown in Fig. 9 (inset) the cytotoxicity of empty SLNs with SA as the core lipid was evaluated in order to confirm any significant loss in cell viability. The results showed negligible cytotoxicity with $90 \%$ cell viability at $3 \mathrm{mg} / \mathrm{ml}$ of SLN dispersions.

The effect of increase in RTA concentration on cell viability was assessed by the MTT assay (Fig. 10). It was found that with RTA-SLNs the toxic effect was dose dependent. At the lowest drug concentration tested of $12.5 \mu \mathrm{g} / \mathrm{ml}$ cell viability decreased to $88.6 \%$ while remaining significantly higher in control cells treated with SLN formulation only $(P<0.05)$. With an increasing drug concentration the cytotoxic effect became even more pronounced. At a drug concentration of $100 \mu \mathrm{g} / \mathrm{ml}$ a significant difference in cell viability was observed between RTA-SLNs and blank SLN formulations, as the cell viability was reduced to $46.8 \%$ $(\mathrm{P}<0.001)$.At an even greater RTA concentration of $200 \mu \mathrm{g} / \mathrm{ml}$ the cell viability was $35.2 \%$. The effect of treatment time on the cytotoxicity of RTA-SLN was also investigated. After being exposed to cells for $48 \mathrm{hr}$ the RTA-SLNs were significantly more effective than after treatment for $24 \mathrm{hr}$, as the cell viability was $19.8 \%$ at an RTA concentration of $200 \mu \mathrm{g} / \mathrm{ml}$. The time dependent increase in the cytotoxic effect suggests that more drug is released from the lipid matrix indicating sustained release effect of RTA-SLN. These findings also show that free RTA (Fig. 10) is significantly more anti-proliferative than RTA-SLN. As the blank SLNs do not induce any significant effect on cell viability, the effect of encapsulated RTA on the LNCap prostate cancer cells was confirmed. 


\subsection{Cellular internalisation and in vitro apoptosis}

SLNs are known to enter cells via endocytosis (Kang et al., 2010). In order to confirm the internalization of the SLN formulation, rhodamine containing SLNs were incubated with LNCap cells and cellular uptake visualised via fluorescent microscopy after staining the nucleus of the cells with DAPI. Internalization of SLN formulations was observed after $24 \mathrm{hr}$. SLN particles were localised in the cytoplasm around the cell nucleus, as illustrated in Fig. 11.

The induction of apoptosis by both pure RTA and within SLNs at different concentrations was detected and quantified by flow cytometry (Fig. 12). Cells treated with pure RTA $(25 \mu \mathrm{g} / \mathrm{ml})$ showed that $24.3 \%$ of the whole population was early apoptotic while $20 \%$ was late apoptotic/ early necrotic. Similarly, when treated with RTA-SLN at $25 \mu \mathrm{g} / \mathrm{ml}$ $27.4 \%$ were early apoptotic and $17.7 \%$ were late apoptotic/ early necrotic. These results suggest that like pure RTA, RTA loaded SLN's also induced apoptosis. At higher concentrations of both treatments an increase in the fraction of late apoptotic/early necrotic cells was found (Fig. 12).

The increase in the percentage of late apoptotic/early necrotic cells following treatment clearly linked RTA and RTA loaded SLNs which appeared to be significantly higher when compared to untreated cells $(P<0.05)$. This increasing population of late apoptotic/early necrotic cells with higher concentration confirmed that enhanced cytotoxicity of RTA-SLN's was dose dependant. Experiments with control (blank SLN treated cells) and untreated cells showed negligible effects in terms of inducing the apoptotic pathways, as no significant alteration were observed in both early apoptotic and late apoptotic/early necrotic cell populations.

\section{CONCLUSIONS}

In the current study SLN dispersions were prepared by HPH and loaded with the anticancer agent RTA. Drug loaded SLNs presented good stability only when SA was used as a core lipid. The dispersions showed high EE with biphasic release where the initial burst release was followed by a sustained pattern. The anticancer activity of RTA-SLNs was assessed in LNCaP prostate cancer cells and showed a viability of $19.8 \%$ at an RTA concentration of $200 \mu \mathrm{g} / \mathrm{ml}$ after a $48 \mathrm{hr}$ incubation. Moreover, fluorescent microscopy images confirmed the internalisation of SLNs inside the cytoplasm of the cell. In vitro apoptosis analysis revealed that upon treating LNCaP cells with RTA-SLNs, $67 \%$ of the cells 
were late apoptotic/early necrotic at a concentration of RTA of $150 \mu \mathrm{g} / \mathrm{ml}$ RTA. Overall the optimized RTA-SLN dispersions appear to be a promising drug delivery system for cancer treatment.

\section{References}

Battaglia, L., Gallarate, M., 2012. Lipid nanoparticles: state of the art new preparation methods and challenges in drug delivery. Exp. Opin. Drug Deliv. 9, 497-508.

Carneiro, G., Silva, E. L., Pacheco, L. A., de Souza-Fagundes, E. M., Corrêa, N. C. R., de Goes, A. M., Ferreira, L. A. M. (2012). Formation of ion pairing as an alternative to improve encapsulation and anticancer activity of all-trans retinoic acid loaded in solid lipid nanoparticles. Int. J. Nanomed. 7, 6011.

Castelli F, Puglia C, Sarpietro M. G. (2005) Characterization of indomethacin-loaded lipid nanoparticles by differential scanning calorimetry. Int. J. Pharm. 304:231-38.

Chen, D. B., Yang, T. Z., Lu, W. L., Zhang, Q. (2001). In vitro and in vivo study of two types of long-circulating solid lipid nanoparticles containing paclitaxel. Chem. Pharm. Bull. 49(11), 1444-1447.

Chinsriwongkul, A., Chareanputtakhun, P., Ngawhirunpat, T., Rojanarata, T., Silaon, W., Ruktanonchai, U., Opanasopit, P. (2012). Nanostructured lipid carriers (NLC) for parenteral delivery of an anticancer drug. AAPS PharmSciTech, 13(1), 150-158.

Fang, J., Chen, S. J., Tong, J. H., Wang, Z. G. (2002). Treatment of Acute Promyelocytic Leukemia with ATRA and As2O3: A Model of Molecular. Cancer Biol. Ther. 1(6), 614-620.

Gasco, M. R. (1993). U.S. Patent No. 5,250,236. Washington, DC: U.S. Patent and Trademark Office.

Göppert TM, Müller RH. (2005) Adsorption kinetics of plasma proteins on solid lipid nanoparticles for drug targeting. Int. J. Pharm.302:172-86.

Jung, H., Kim, J., Park, J., Lee, S., Lee, H., Kuboi, R., Kawai, T. (2006). Atomic force microscopy observation of highly arrayed phospholipid bilayer vesicle on a gold surface. J. Biosci. Bioeng. 102(1), 28-33. 
Kang, K. W., Chun, M. K., Kim, O., Subedi, R. K., Ahn, S. G., Yoon, J. H., Choi, H. K. (2010). Doxorubicin-loaded solid lipid nanoparticles to overcome multidrug resistance in cancer therapy. Nanomed: Nanotech. Biol. Med. 6(2), 210-213.

Li, W. W., Li, V. W., Hutnik, M., Chiou, A. S. (2011). Tumour angiogenesis as a target for dietary cancer prevention. J. Oncol. 2012.

Lim, S. J., Kim, C. K. (2002). Formulation parameters determining the physicochemical characteristics of solid lipid nanoparticles loaded with all-trans retinoic acid. Int. J. Pharm. 243(1), 135-146.

Liu, J., Gong, T., Wang, C., Zhong, Z., Zhang, Z. (2007). Solid lipid nanoparticles loaded with insulin by sodium cholate-phosphatidylcholine-based mixed micelles: preparation and characterization. Int. J. Pharm. 340(1), 153-162.

Maden, M. (2007). Retinoic acid in the development, regeneration and maintenance of the nervous system. Nat. Rev. Neurosci. 8(10), 755-765.

McCormick, D. L., Rao, K. V. N., Steele, V. E., Lubet, R. A., Kelloff, G. J., Bosland, M. C. (1999). Chemoprevention of rat prostate carcinogenesis by 9-cis-retinoic acid. Cancer Res. $59(3), 521-524$.

Mehnert, W., Mäder, K. (2001). Solid lipid nanoparticles: production, characterization and applications. Adv. Drug Deliv. Rev. 47(2), 165-196.

Mueller, R. H., Maeder, K., Gohla, S. (2000). Solid lipid nanoparticles (SLN) for controlled drug delivery-a review of the state of the art. Eur. J. Pharm. Biopharm. 50(1), 161-177.

Muindi, J., Frankel, S., Miller, W. J., Jakubowski, A., Scheinberg, D. A., Young, C., Warrell, R. J. (1992). Continuous treatment with all-trans retinoic acid causes a progressive reduction in plasma drug concentrations: implications for relapse and retinoid" resistance" in patients with acute promyelocytic leukemia. Blood, 79(2), 299-303.

Mulik, R. S., Mönkkönen, J., Juvonen, R. O., Mahadik, K. R., Paradkar, A. R. (2012). Apoptosis-induced anticancer effect of transferrin-conjugated solid lipid nanoparticles of curcumin. Cancer Nanotechnol., 3(1-6), 65-81.

Müller RH, Keck CM. (2004) Challenges and solutions for the delivery of biotech drugs - a review of drug nanocrystal technology and lipid nanoparticles. J. Biotech. 
Müller, R.H., Shegokar, R., Keck, C.M., 2011. 20 years of lipid nanoparticles (SLN and NLC): present state of development and industrial applications. Curr. Drug Discov. Technol. $8(3), 207-227$.

Nayak, A. P., Tiyaboonchai, W., Patankar, S., Madhusudhan, B., Souto, E. B. (2010). Curcuminoid-loaded lipid nanoparticles: novel approach towards malaria treatment. Coll. Surf. B: Biointerfaces 81(1), 263-273.

Orlandi, M., Mantovani, B., Ammar, K., Avitabile, E., Dal Monte, P., Bartolini, G. (2002). Retinoids and cancer: antitumoural effects of ATRA, 9-cis RA and the new retinoid IIF on the HL-60 leukemic cell line. Medical principles and practice: Int. J. Kuwait Univ. Health Sci. Centre, 12(3), 164-169.

Padhye, S. G., Nagarsenker, M. S. (2013). Simvastatin solid lipid nanoparticles for oral delivery: Formulation development and In vivo evaluation. Ind. J. Pharm. Sci. 75(5), 591.

Potta, S. G., Minemi, S., Nukala, R. K., Peinado, C., Lamprou, D. A., Urquhart, A., Douroumis, D. (2011). Preparation and characterization of ibuprofen solid lipid nanoparticles with enhanced solubility. J. Microencapsul. 28(1), 74-81.

Serpe, L., Catalano, M. G., Cavalli, R., Ugazio, E., Bosco, O., Canaparo, R., Zara, G. P. (2004). Cytotoxicity of anticancer drugs incorporated in solid lipid nanoparticles on HT-29 colorectal cancer cell line. Eur. J. Pharm. Biopharm. 58(3), 673-680.

Siekmann, B., Westesen, K. (1994). Thermoanalysis of the recrystallization process of melthomogenized glyceride nanoparticles. Coll. Surf. B: Biointerf. 3(3), 159-175.

Speth, P. A. J., Van Hoesel, Q. G. C. M., Haanen, C. (1988). Clinical pharmacokinetics of doxorubicin. Clinical Pharmacokin. 15(1), 15-31.

Sun, J., Bi, C., Chan, H. M., Sun, S., Zhang, Q., Zheng, Y. (2013). Curcumin-loaded solid lipid nanoparticles have prolonged in vitro antitumour activity, cellular uptake and improved in vivo bioavailability. Coll. Surf. B: Biointerf. 111, 367-375.

Tiyaboonchai, W., Tungpradit, W., Plianbangchang, P. (2007). Formulation and characterization of curcuminoids loaded solid lipid nanoparticles. Int. J. Pharm. 337(1), 299306. 
Yang, X., Liu, Y., Liu, C., Zhang, N. (2012). Biodegradable solid lipid nanoparticle flocculates for pulmonary delivery of insulin. J. Biomed. Nanotechnol. 8(5), 834-842.

Yang, M., He, S., Fan, Y., Wang, Y., Ge, Z., Shan, L., Gao, C. (2014). Microenvironmental $\mathrm{pH}$-modified solid dispersions to enhance the dissolution and bioavailability of poorly watersoluble weakly basic GT0918, a developing anti-prostate cancer drug: Preparation, characterization and evaluation in vivo. Int. J. Pharm. 475(1), 97-109.

Yousefi, A., Esmaeili, F., Rahimian, S., Atyabi, F., Dinarvand, R. (2009). Preparation and in vitro evaluation of a pegylated nano-liposomal formulation containing docetaxel. Sci. Pharm. 77, 453-464. 
Table 1: Blank SLN dispersions made by using TS, SA, PR and TL ( $n=3)$.

\begin{tabular}{|l|l|c|c|c|c|c|}
\hline SLNs & \multicolumn{1}{|c|}{ Lipid } & $\begin{array}{c}\text { Lipid } \\
(\mathbf{m g})\end{array}$ & $\begin{array}{c}\text { P188 } \\
(\mathbf{m g})\end{array}$ & $\begin{array}{c}\text { Particle size } \\
(\mathbf{n m}, \pm \mathbf{S D})\end{array}$ & $\begin{array}{c}\text { Zeta potential } \\
(\mathbf{m V}, \pm \text { SD })\end{array}$ & $\begin{array}{c}\text { PI } \\
( \pm \text { SD })\end{array}$ \\
\hline BL- TS & Tristearin & 500 & 250 & $148.4 \pm 3.1$ & $-19.20 \pm 1.61$ & $0.11 \pm 0.03$ \\
\hline BL- SA & Stearic acid & 500 & 250 & $145.1 \pm 2.7$ & $-18.74 \pm 1.77$ & $0.10 \pm 0.02$ \\
\hline BL- PR & Precirol & 500 & 250 & $143.4 \pm 1.2$ & $-15.47 \pm 0.13$ & $0.08 \pm 0.03$ \\
\hline BL- TL & Trilaurin & 500 & 250 & $149.2 \pm 5.5$ & $-13.32 \pm 0.23$ & $0.07 \pm 0.01$ \\
\hline
\end{tabular}

Table 2: RTA loaded SLN dispersions made using SA and TS $(n=3)$.

\begin{tabular}{|c|c|c|c|c|c|c|c|}
\hline SLNs & Lipid & $\begin{array}{c}\text { Lipid } \\
(\mathbf{m g})\end{array}$ & $\begin{array}{c}\text { P188 } \\
(\mathbf{m g})\end{array}$ & $\begin{array}{c}\text { RTA } \\
(\mathbf{m g})\end{array}$ & $\begin{array}{c}\text { Particle size } \\
(\mathbf{n m}, \pm \text { SD })\end{array}$ & $\begin{array}{c}\text { Zeta potential } \\
(\mathbf{m V}, \pm \text { SD })\end{array}$ & $\begin{array}{c}\text { PI } \\
( \pm \text { SD })\end{array}$ \\
\hline RTA- TS & Tristearin & 500 & 250 & 50 & $255.9 \pm 2.7$ & $-0.06 \pm 0.01$ & $0.30 \pm 0.09$ \\
\hline RTA- SA & Stearic acid & 500 & 250 & 50 & $232.3 \pm 3.1$ & $-8.55 \pm 1.71$ & $0.21 \pm 0.05$ \\
\hline
\end{tabular}




\section{FIGURES CAPTION LIST}

Fig. 1. (a) RTA-SA SLN dispersions. (b) Particle size distribution of RTA-SA SLNs (where the size quoted is the $\mathrm{z}$-average mean $(\mathrm{nm})$ of the hydrodynamic diameter $(\mathrm{nm})$ of the lipid dispersion. (c) Zeta potential (mV) of RTA-SA SLNs.

Fig. 2. (a) RTA-TS SLN dispersions. (b) Particle size distribution of RTA-TS SLNs (where the size quoted is the $\mathrm{z}$-average mean $(\mathrm{nm})$ of the hydrodynamic diameter $(\mathrm{nm})$ of the lipid dispersion. (c) Zeta potential (mV) of RTA-TS SLNs.

Fig. 3. Transmission electron micrographs of A) BL- SLNs and B) RTA-SLNs.

Fig. 4. AFM images of blank-SLNs (A, B) and RTA loaded SLNs (C, D).

Fig. 5. XRD diffractograms of bulk SA, poloxamer 188 and RTA.

Fig. 6. XRD diffractograms of blank SLN, RTA-SLNs and RTA.

Fig. 7. DSC thermograms of RTA, SA, P188, BL-SLNs and RTA-SLNs.

Fig. 8. Drug release profile of SA based SLNs loaded with RTA.

Fig. 9. a) Anti-proliferative effects of pure RTA on prostate cancer cells (LNCap) using the MTT assay for a $24 \mathrm{hr}$ incubation and b) cytotoxicity of stearic acid based blank SLNs (BLSLNs), after 24 hours of incubation (inset).

Fig. 10. Cytotoxicity of stearic acid based RTA loaded SLN (RTA-SLN), after 24 and $48 \mathrm{hr}$ incubation. Data is represented as mean \pm S.D. $(\mathrm{n}=3) . P<0.05, * P<0.0001$, RTA-SLN (24 hr) vs. empty SLN and $P<0.05, * * P<0.0001$, RTA-SLN (48 hr) vs. empty SLN.

Fig. 11. Fluorescent images of the cellular uptake of empty formulated SLN localisation in the cytoplasm of the cell. The nucleus of the cell was stained blue with DAPI and the SLN formulation was labelled with rhodamine.

Fig. 12. Quantitative apoptotic measurement in LNCap cells after treatment with BL-SLN (blank SLN), pure RTA and RTA-SLN (RTA loaded SLN). (A) Dose dependent effect on early apoptosis by treatment with a concentration of 25,75 and $150 \mu \mathrm{g} / \mathrm{ml}$ of RTA-SLNs for $48 \mathrm{hr}$, determined by flow cytometry analysis. (B) Dose dependent effect on late apoptosis by treatment with a concentration of 25,75 and $150 \mu \mathrm{g} / \mathrm{ml}$ of RTA-SLNs for $48 \mathrm{hr}$, determined by flow cytometry analysis. The results are expressed as bar chart. Data as mean \pm S.D., $(\mathrm{n}=$ 3). (*) $P<0.05$, Control versus RTA-SLN $(25 \mu \mathrm{g} / \mathrm{ml})$, (**) $P<0.05$, Control versus RTASLN $(75 \mu \mathrm{g} / \mathrm{ml}),(* * *) P<0.05$, Control versus RTA-SLN $(150 \mu \mathrm{g} / \mathrm{ml})$ (C) Dose dependent effects are expressed as dot plot of PE AnnexinV versus 7-AAD. Top left: necrotic cells; top right: late apoptotic cells/early necrotic cells; bottom left: live cells; and bottom right: early apoptotic cells. 
a

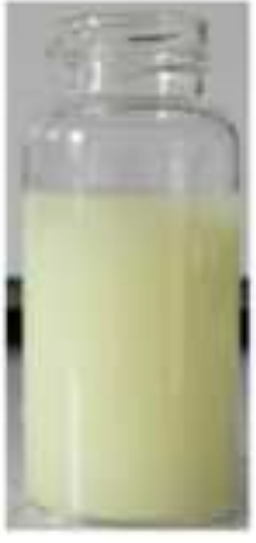

Setbahionir int

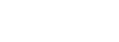

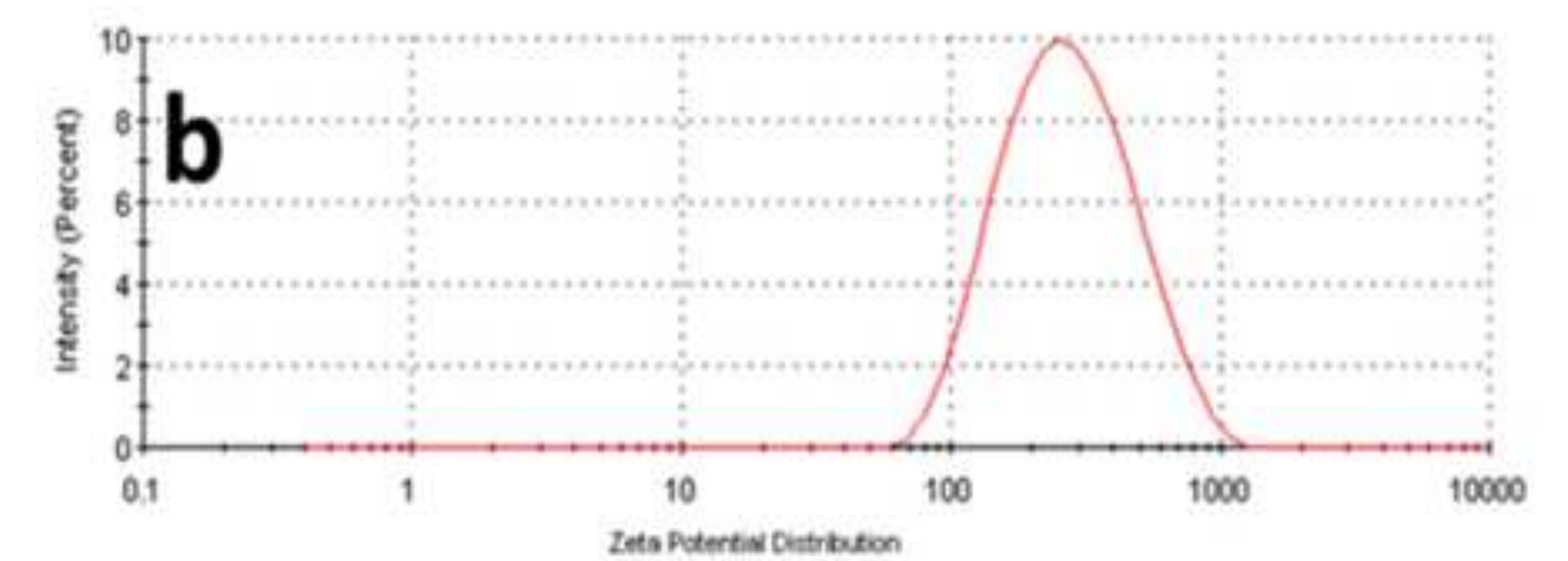

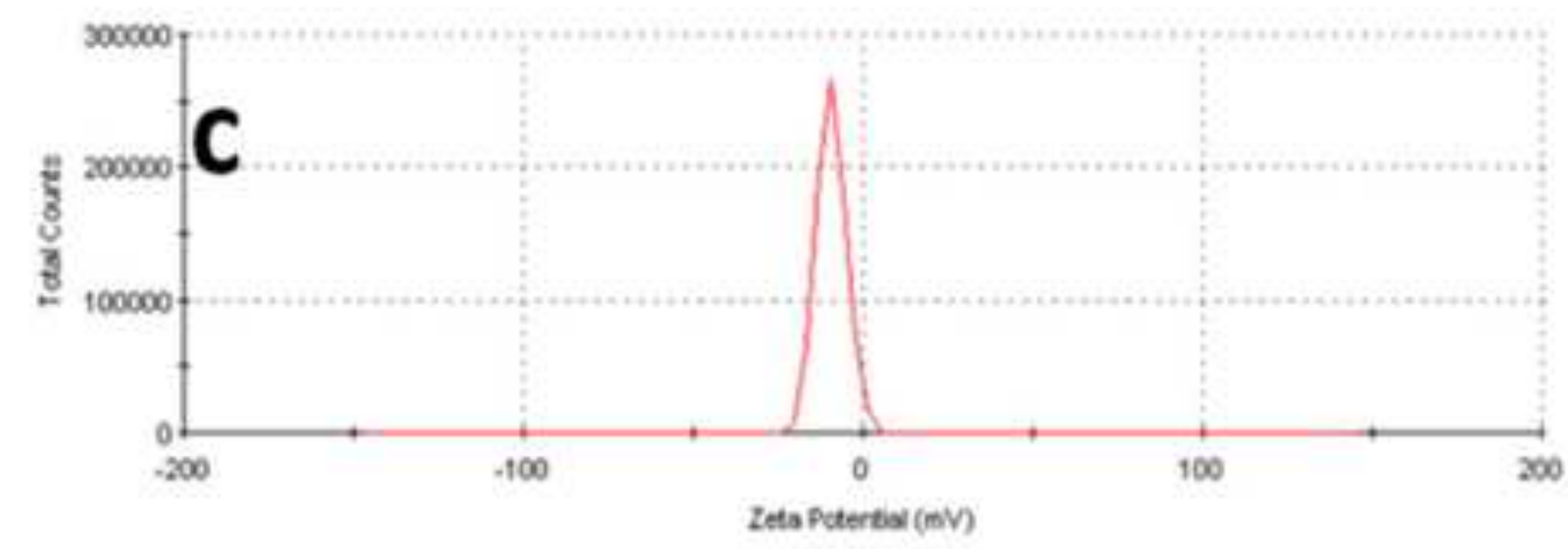

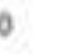

(



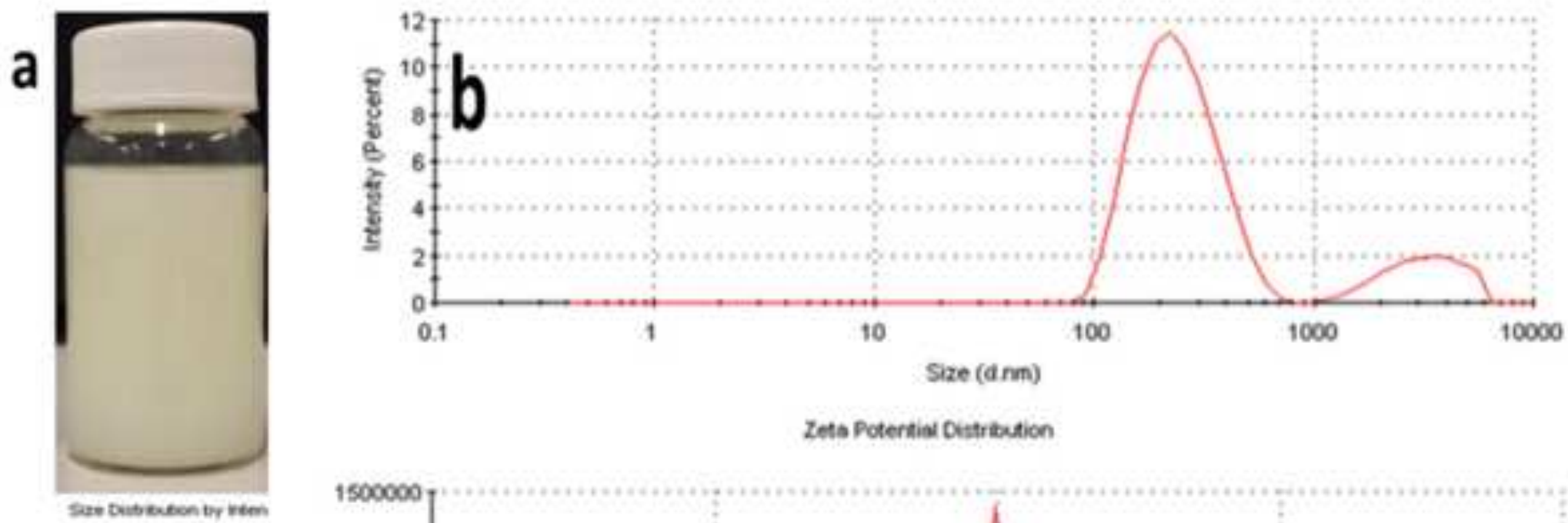

Zeto Potertial Oustratation

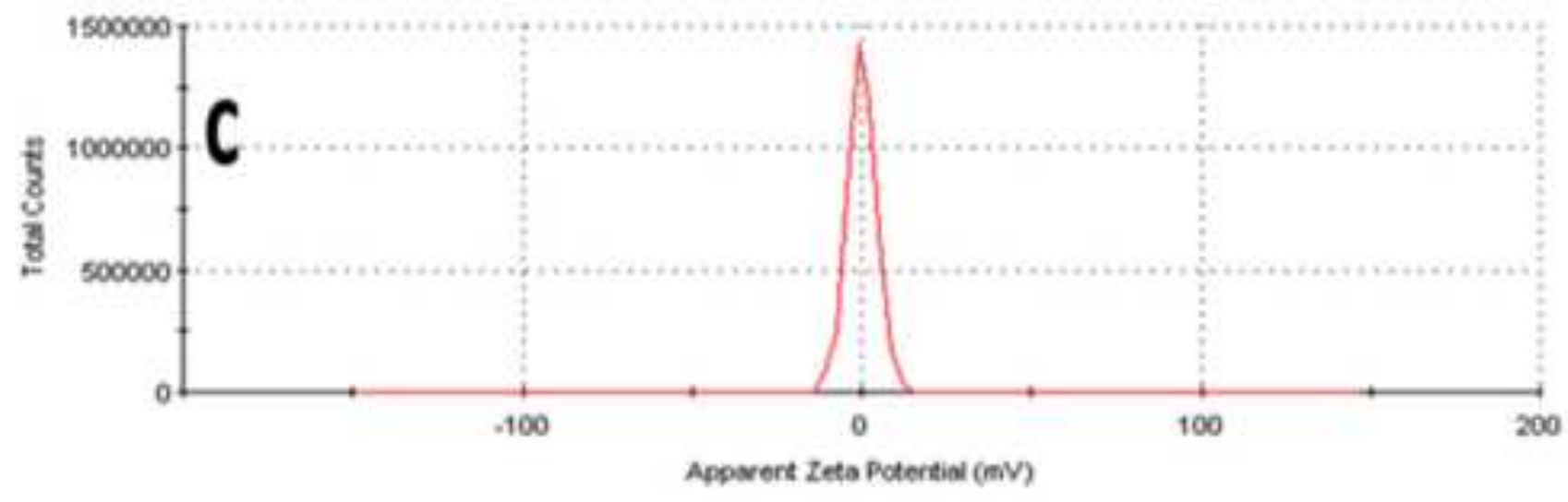




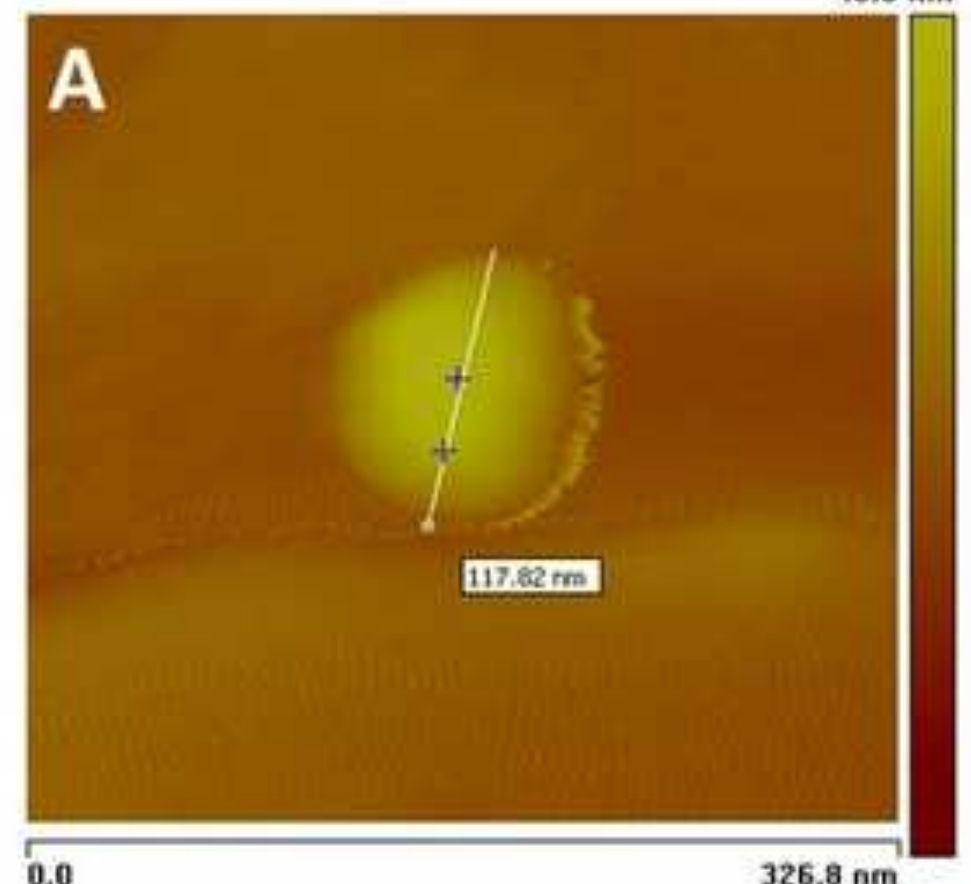

$326.8 \mathrm{~nm}$
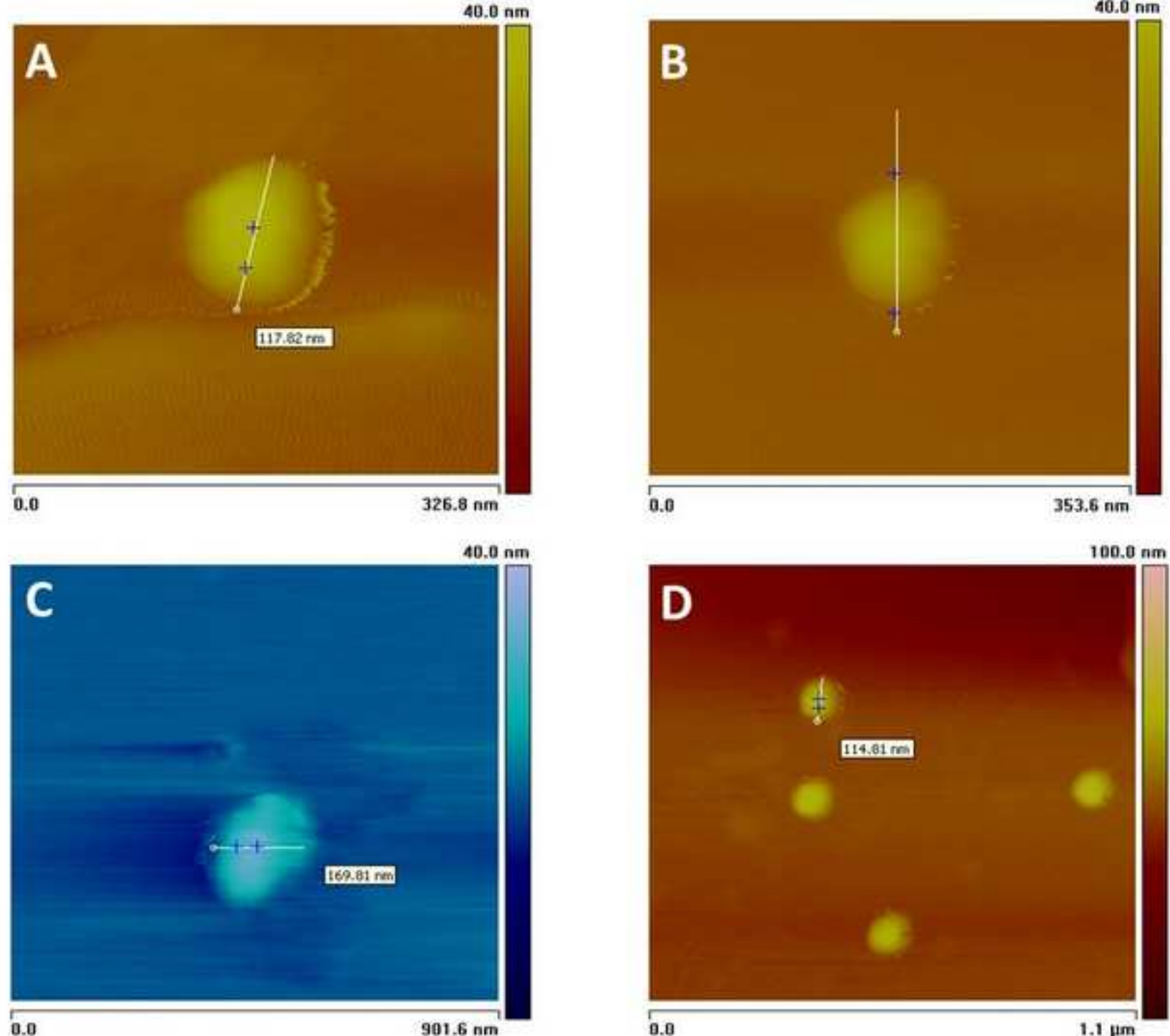

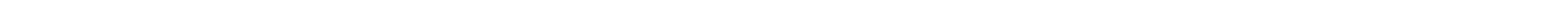

(s)
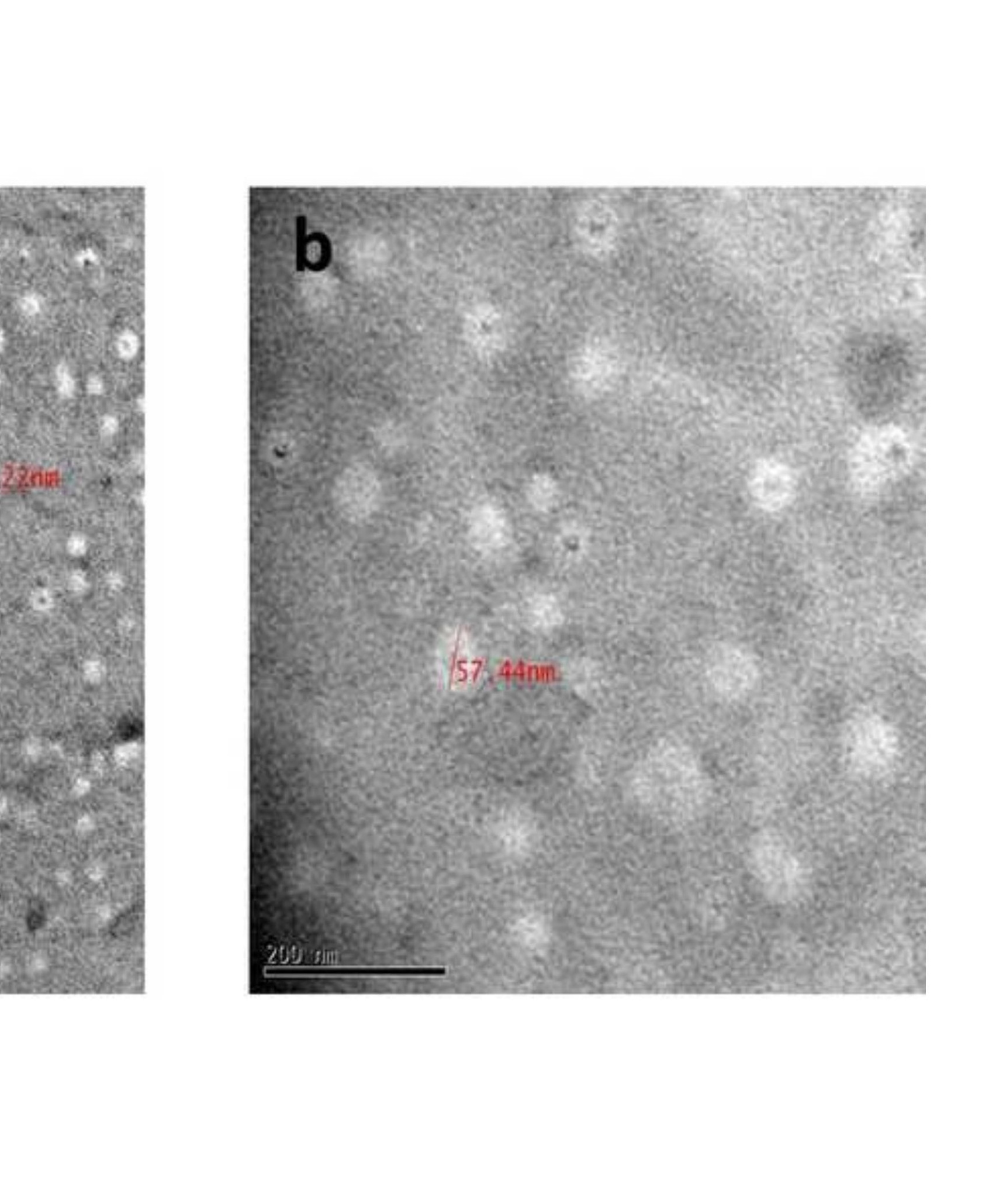


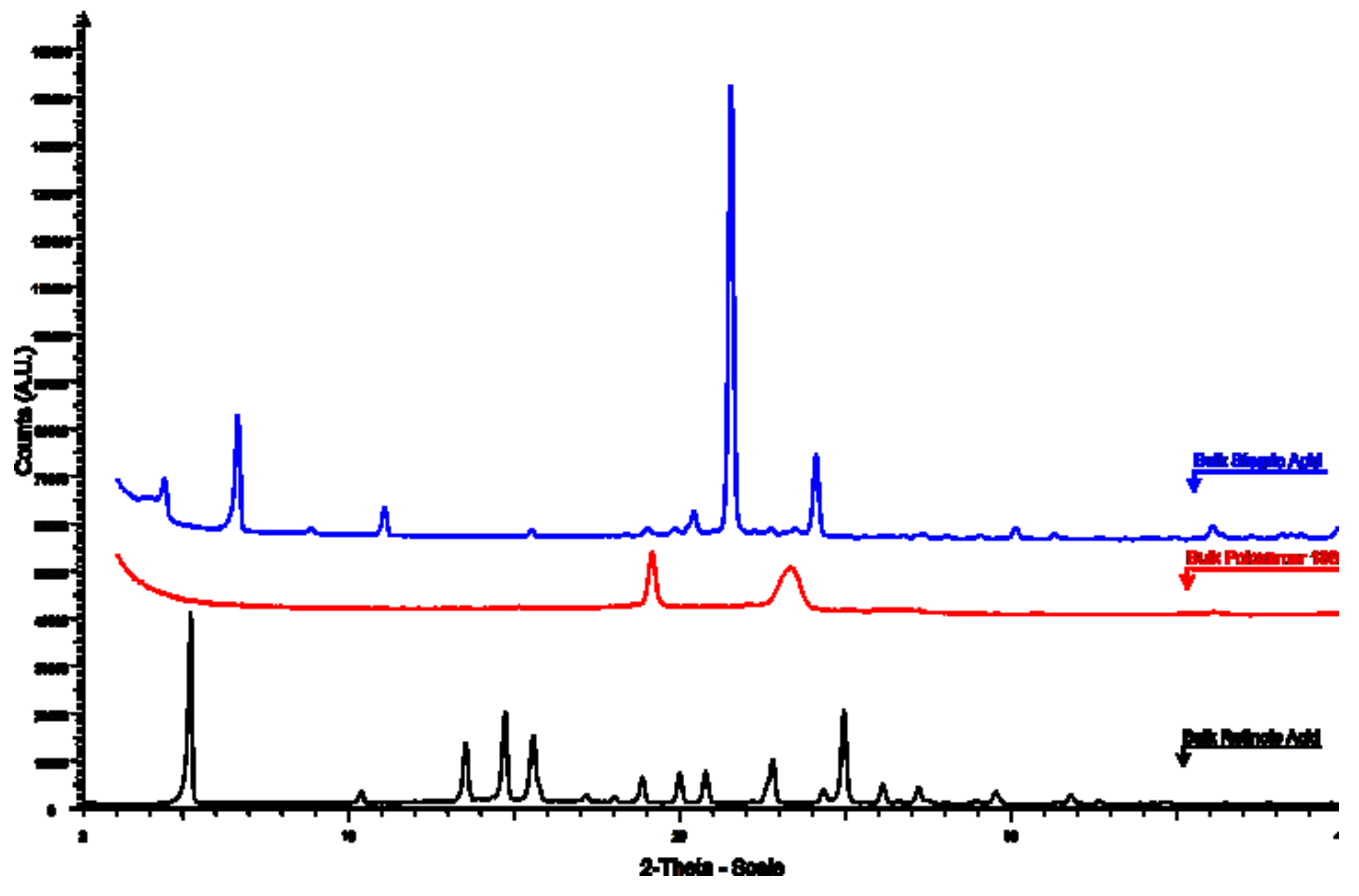




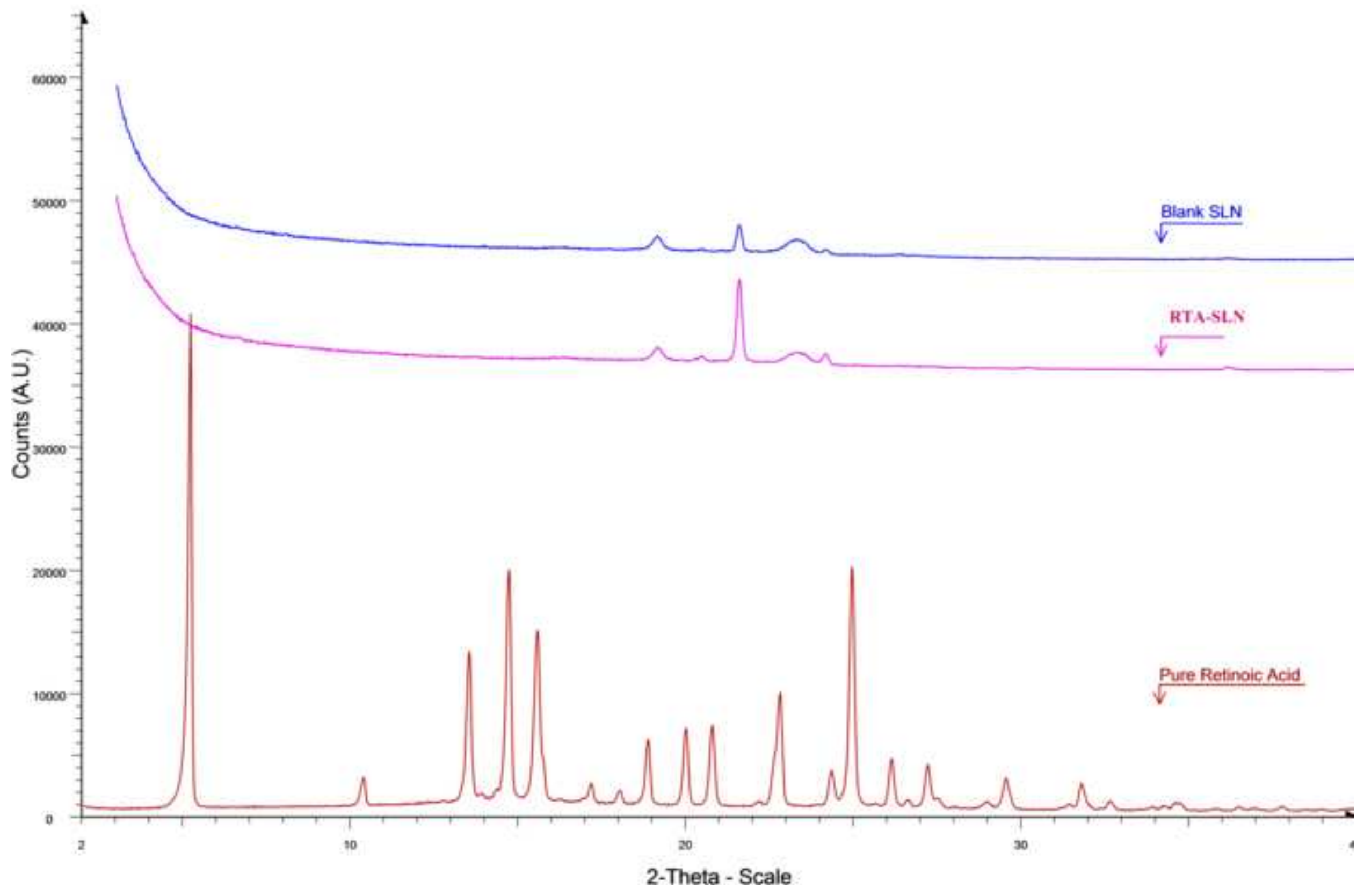




\section{Aexo}

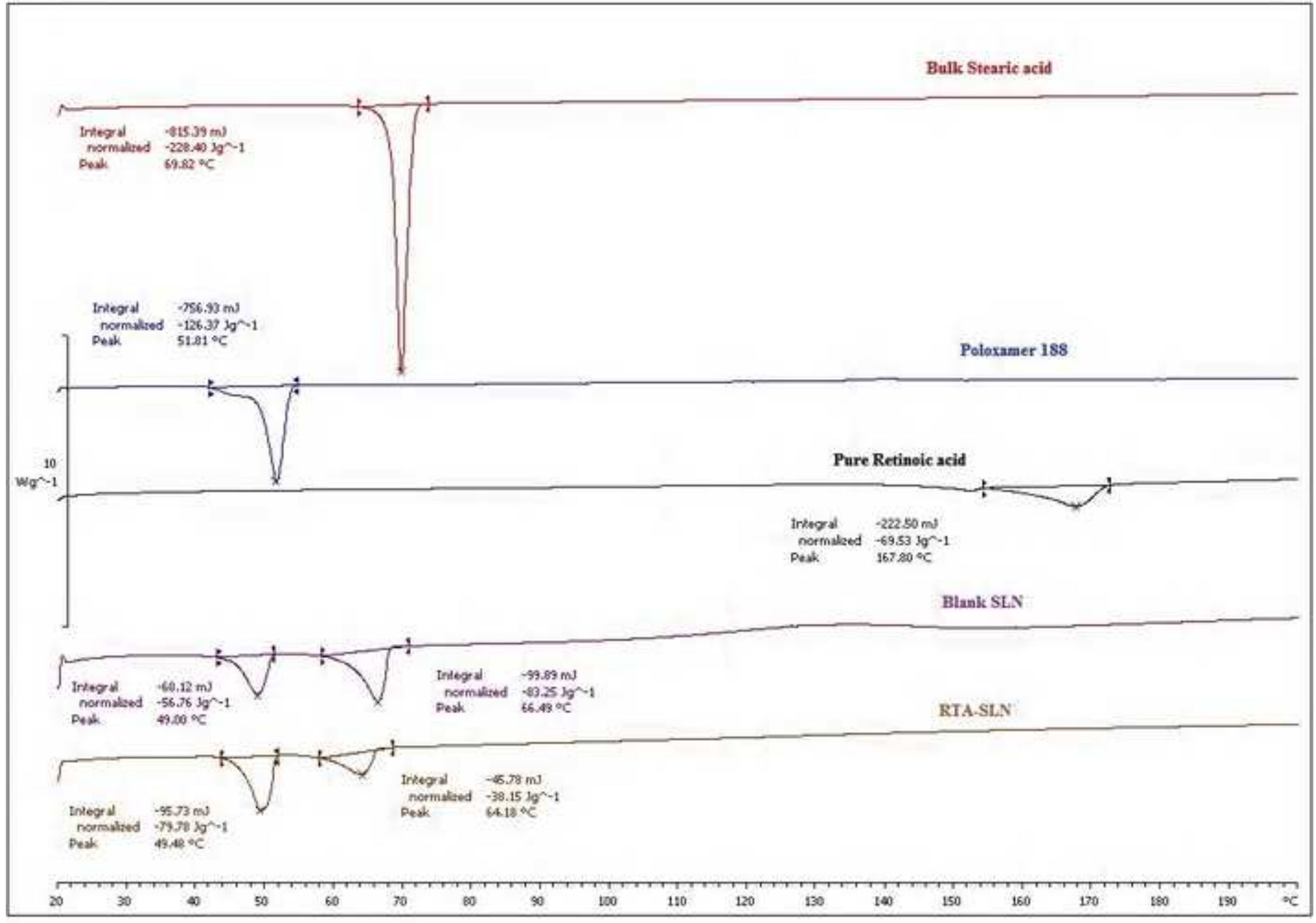


Figure(s)

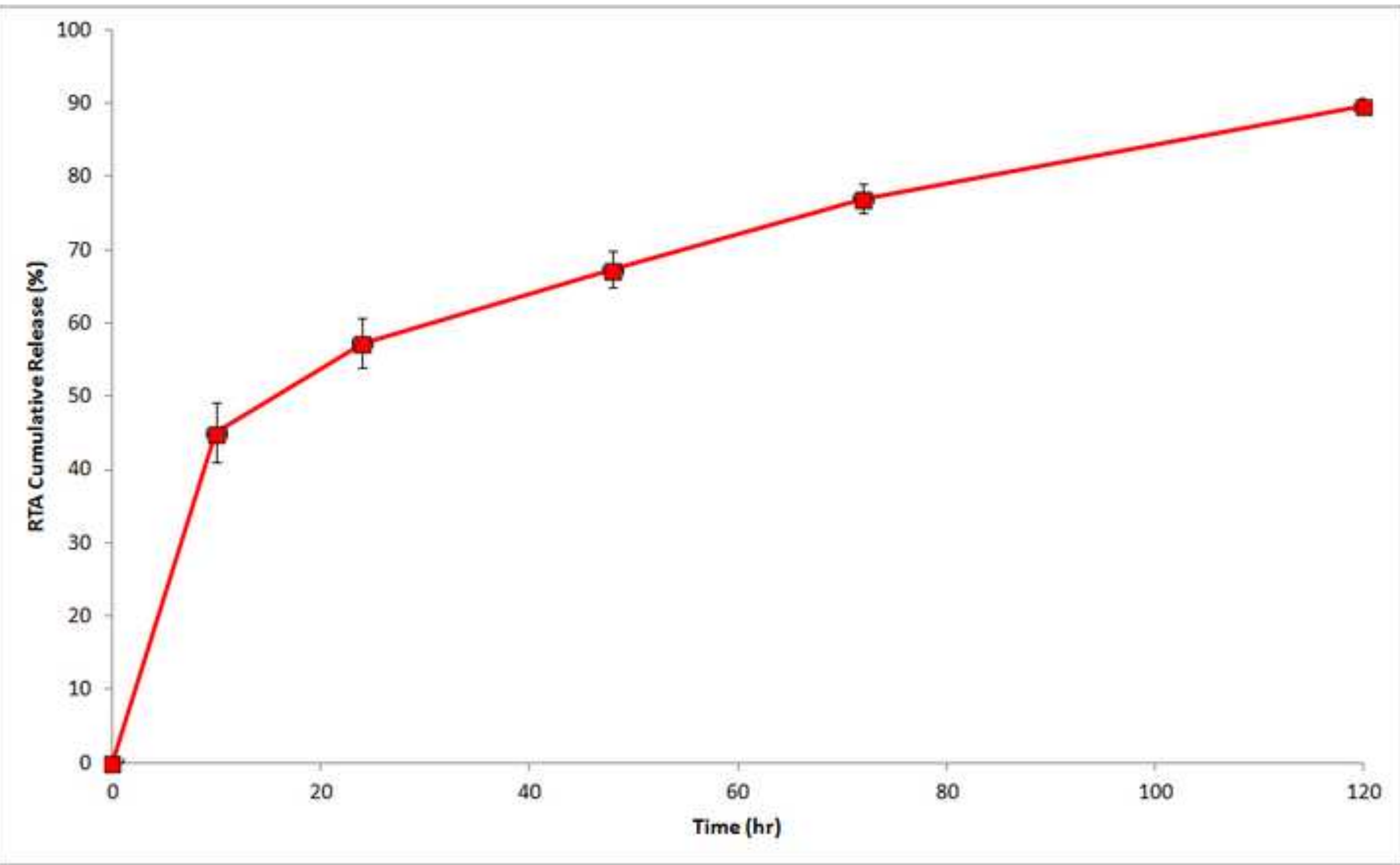


Figure(s

\author{
Fis)
}

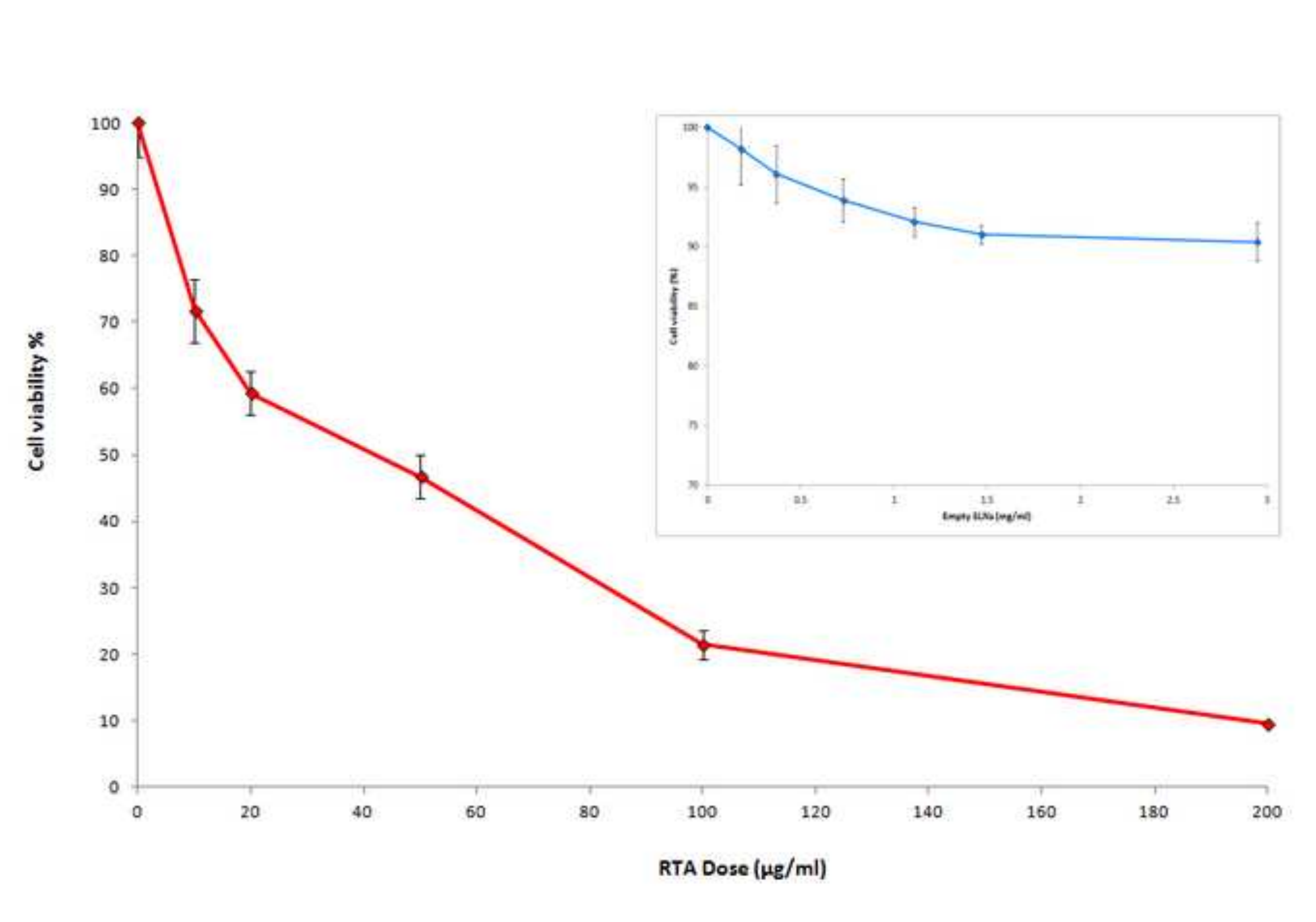

RTA Dose $(\mu \mathrm{g} / \mathrm{ml})$

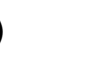

00

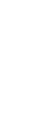
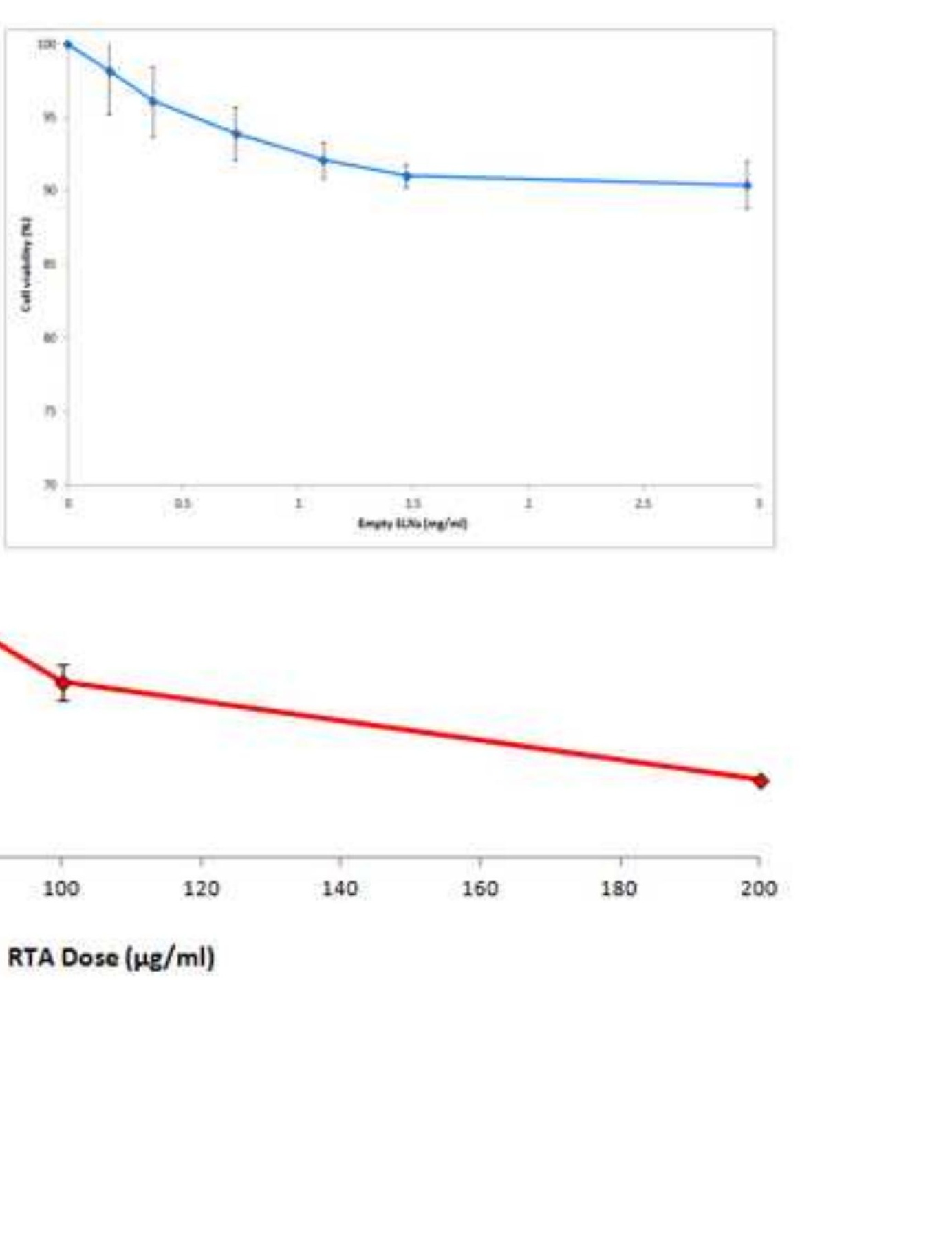


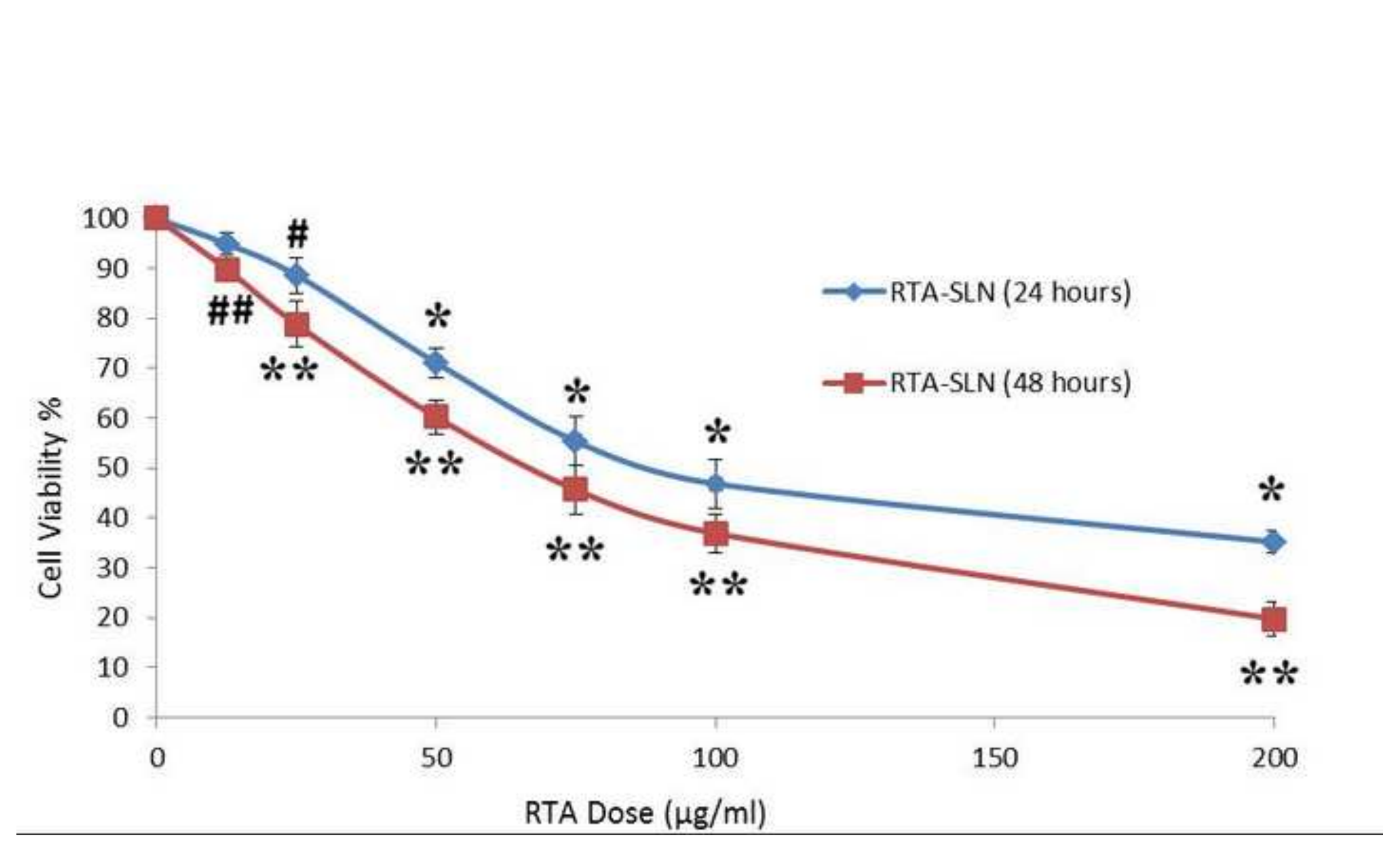

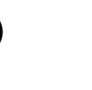

\section{.}
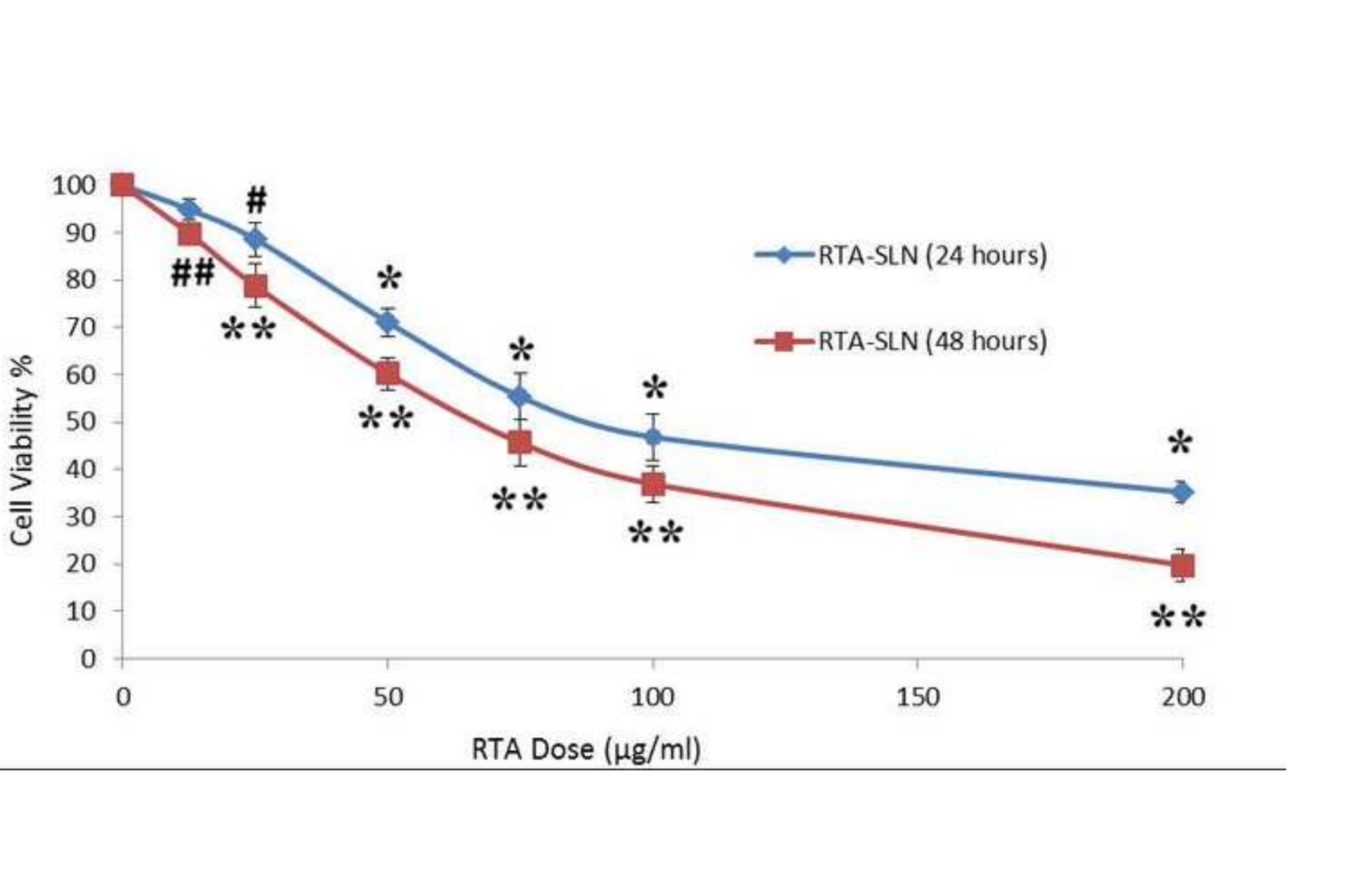

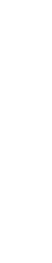

.

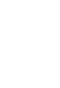

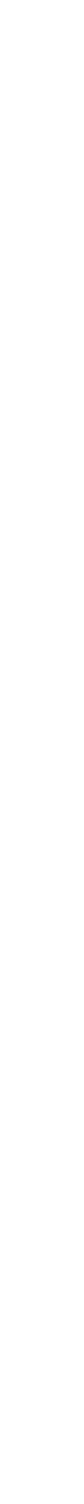




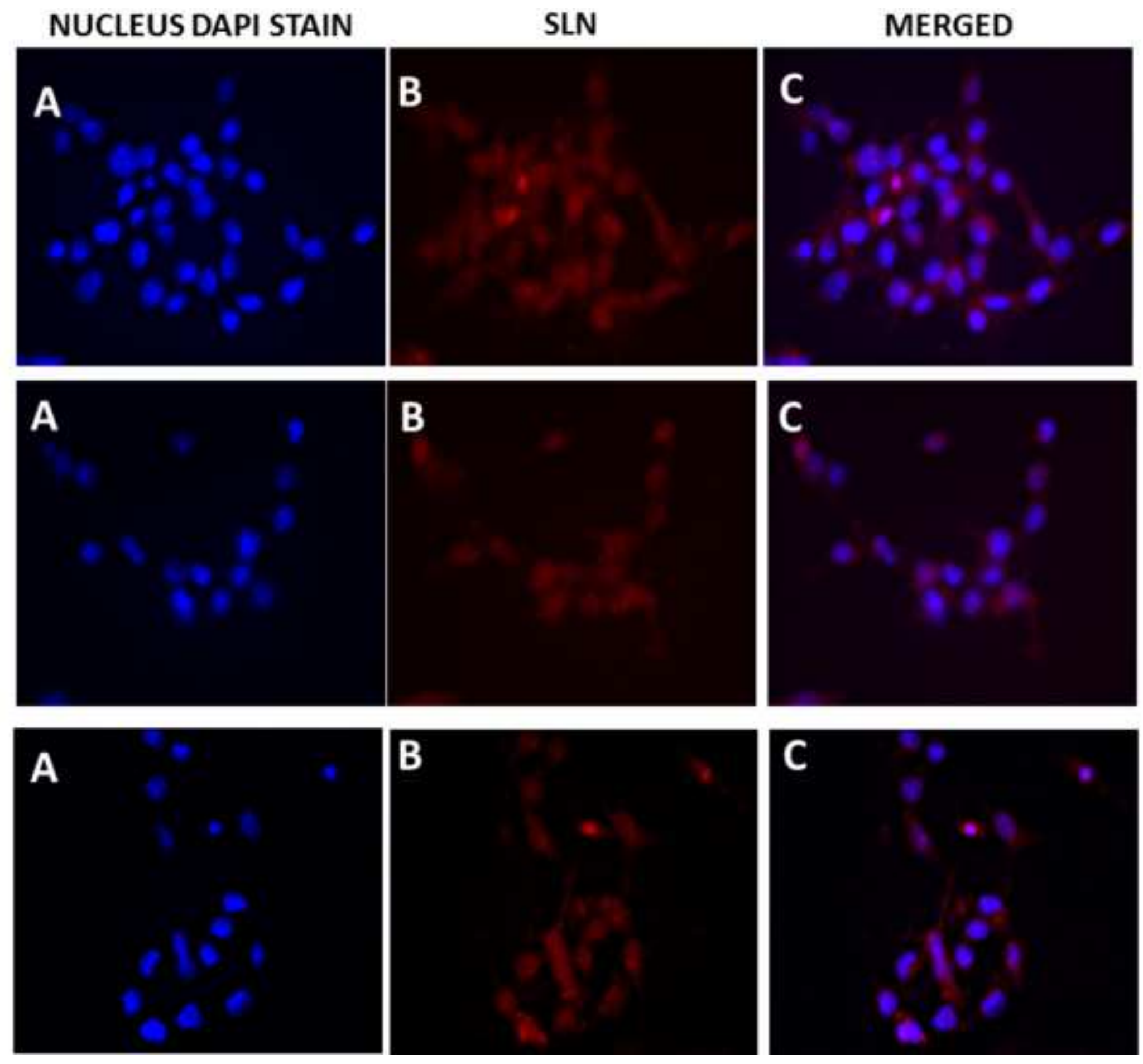



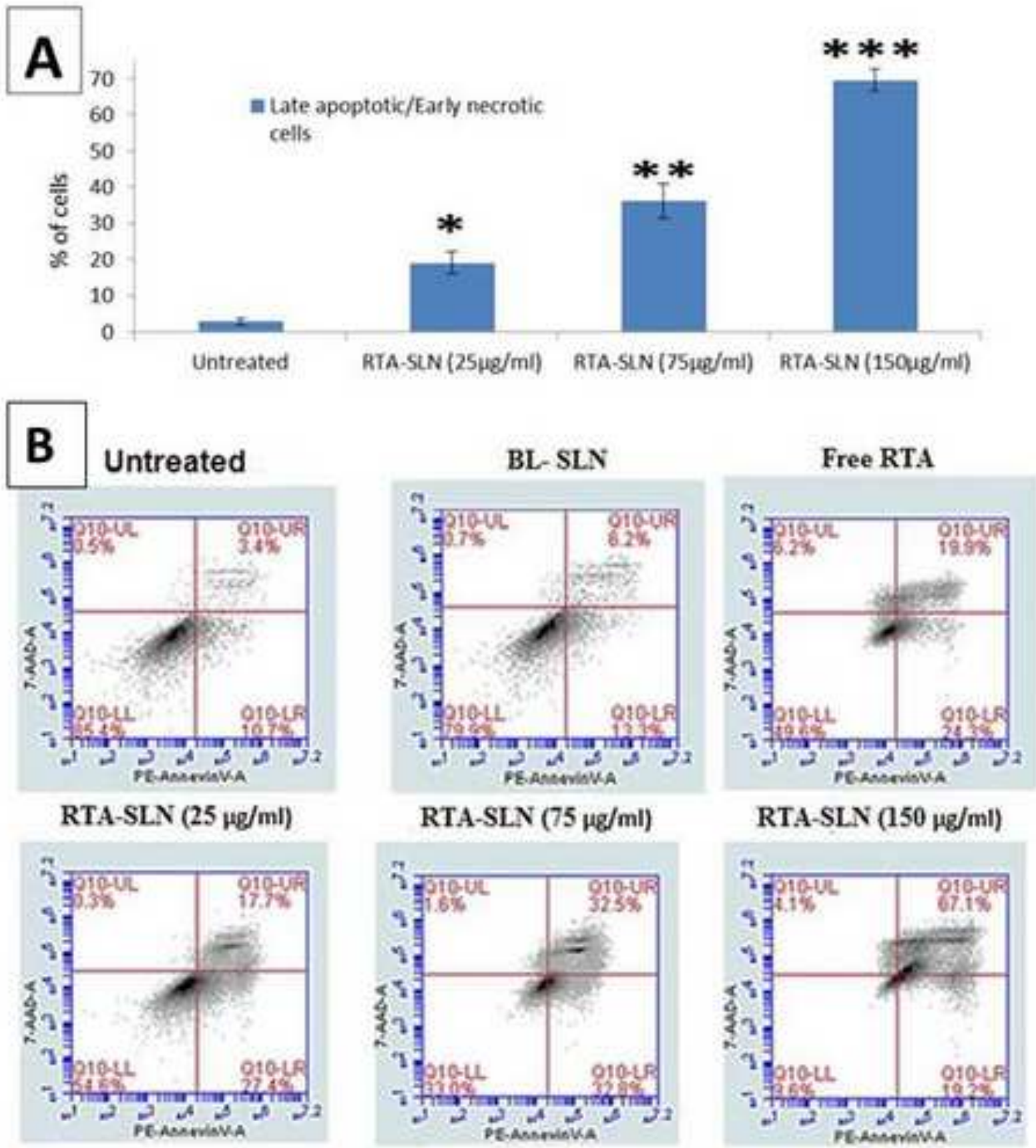